\title{
On Regression Analysis of Spatial Proportional Data with Zero/One Values
}

\author{
Xiaoping Feng ${ }^{\mathrm{a}}$, Jun Zhu ${ }^{\mathrm{b}}$, Michelle M. Steen-Adams ${ }^{\mathrm{c}}$ \\ ${ }^{a}$ Department of Statistics, University of Wisconsin, Madison, WI 53706 \\ ${ }^{b}$ Department of Statistics and Department of Entomology, University of Wisconsin, Madison, WI 53706 \\ ${ }^{c}$ Department of Environmental Studies, University of New England, Biddeford, ME 04005
}

\begin{abstract}
Proportions including exact zero and/or one values observed at spatial locations in a study area are often encountered in environmental and ecological studies. In this paper, we propose a new spatial beta-Bernoulli mixture model that combines a beta distribution and a Bernoulli distribution. The beta component links the original response on the open unit interval $(0,1)$ to covariates via regression and is flexible to capture a variety of shapes of the data distributions. Further, the Bernoulli component models the probability of zero/one values with regression. In addition, we propose a novel spatial generalized Tobit model which extends an existing spatial Tobit model by applying an inverse beta cumulative distribution function transformation. A composite likelihood approach is developed for parameter estimation by maximizing a pairwise likelihood function for each model. The standard errors of the parameter estimates are obtained via the inverse of the Godambe information matrix. A simulation study is conducted to evaluate the performance of the proposed models and methods, followed by an ecological data example. Connections among the spatial beta-Bernoulli mixture model, the spatial generalized Tobit model, and the spatial Tobit model are explored using both simulated and real data.

Keywords: Beta-Bernoulli mixture model; composite likelihood estimation; Gaussian latent variable; generalized Tobit model; Godambe information; spatial statistics.
\end{abstract}

Email addresses: fengxestat.wisc.edu (Xiaoping Feng), jzhuestat.wisc.edu (Jun Zhu), msteenadams@une.edu (Michelle M. Steen-Adams) 


\section{Introduction}

Of interest is regression analysis of spatial proportional data with zero and/or one values (henceforth, zero/one values). The motivating example is an integrative landscape ecology and environmental history study that aims to assess the influence of past land-ownership characteristics on landscape structure in northern Wisconsin, USA. The raw data consist of proportions of aspen (Populus spp.)-paper birch (Betula papryfera) (APB) aerial extent in each quarter section with about $12 \%$ of zero/one values, corresponding to no or all APB in those quarter sections (Figure 11. An objective of the study is to evaluate the influence of various ownership covariates on APB. Standard linear regression analysis may not be appropriate to relate the response to covariates, as the probability model does not restrict the range of the response to the unit interval and the spatial dependence is not accounted for. The purpose of this paper is to develop new statistical models and their inference for the regression analysis of spatial proportional data with zero/one values.

The family of beta distributions characterized by two shape parameters is flexible for modeling proportions on the open unit interval $(0,1)$, since the density can take on a variety of shapes for different values of the shape parameters. Several recent studies have considered beta regression models. For independent data, Ferrari and Cribari-Neto (2004) proposed a regression model where the response variable follows a beta distribution parameterized by a regression mean and a precision parameter, enabling a more straightforward interpretation of the regression parameters. Simas et al. (2010) extended this model to allow regression for the precision parameter as well. For dependent data, Eskelson et al. (2011) and Guolo and Varin (2014) proposed beta regression models to accommodate spatial dependence and temporal dependence, respectively. However, the aforementioned studies did not consider the possibility of zero/one values in the data.

In practice, the proportional data may contain exact zero/one values and thus are on the closed unit interval $[0,1]$ (or half-closed intervals $[0,1)$ and $(0,1]$ ). For independent data, Ospina and Ferrari (2010, 2012) described a class of models based on a mixture of continuous and discrete distributions with probability mass at zero/one. For spatial proportional data, Feng et al. (2014) 
proposed a spatial Tobit model, and the distribution of zero/one values are handled by the thresholding parameter and the regression mean. Although the spatial Tobit model is a natural extension of a spatial probit model for spatial binary data (Heagerty and Lele, 1998; De Oliveira, 2000), the Gaussian latent variable restricts the distribution to be unimodal in the open interval $(0,1)$, which may not be appropriate for some data distributions in practice (Kieschnick and McCullough, 2003).

Although beta regression models are well established for the open unit interval, it remains a challenge to model spatial proportional data observed on the closed unit interval. We will propose a new spatial beta-Bernoulli mixture model that accommodates exact zero/one values and takes into account spatial dependence in the spatial proportional data using a Gaussian latent process. Compared with the existing spatial Tobit model, the spatial beta-Bernoulli mixture model captures a wider variety of shapes of data distributions on the open interval $(0,1)$. In addition, we extend the spatial Tobit model by applying an inverse beta cumulative distribution function (CDF) transformation on the Gaussian latent variable, which will be referred as a spatial generalized Tobit model. Similar to the spatial Tobit model, the resulting new model is based on the idea of thresholding a spatial latent process, but overcomes the limitation of the Gaussian latent variable. These two new models assume two distinct data generation mechanisms and can be applied to different situations in practice.

Maximum likelihood estimation for our spatial beta-Bernoulli mixture model and spatial generalized Tobit model requires matrix factorization and multiple integration and thus, is not feasible when the sample size is relatively large. Here, we adopt an alternative composite likelihood approach to parameter estimation and inference, where the higher-dimensional full likelihood function is projected to lower-dimensional likelihood components that are more feasible to compute (Rao, 1970, Lindsay, 1988). Unlike the existing spatial Tobit model, both models involve functional transformations related to the inverse beta CDF and closed-form score functions are not straightforward to derive. Thus we use numerical score functions and evaluate the performance of the new models via simulated and real data examples in comparison with the existing spatial Tobit model. 
The remainder of the paper is organized as follows. In Section 2 , we propose a spatial betaBernoulli mixture model. In Section 3, we take a composite likelihood approach to parameter estimation and statistical inference. In Section 4, we propose an alternative spatial generalized Tobit model, by extending the spatial Tobit model with an inverse beta CDF transformation. Section 5 presents a simulation study that evaluates the finite-sample properties of composite likelihood estimation and compares different models. The application to the data example is given in Section 6 , followed by conclusions and a discussion in Section 7.

\section{Spatial Beta-Bernoulli Mixture Model}

For proportional data with zero/one values, we propose a spatial beta-Bernoulli mixture model. Let $N$ denote the number of sampling sites in a study area. For $i=1, \ldots, N$, let $Y_{i}$ denote the response variable at site $i$ that follows a Bernoulli distribution with probability $\eta$ and a beta distribution with probability $1-\eta$, where $\eta \in[0,1]$ determines the probability that $Y_{i}$ follows a Bernoulli distribution.

More specifically, there are two mixture components in the spatial beta-Bernoulli mixture model, one for the Bernoulli distribution and the other for the beta distribution. For the Bernoulli component, we let

$$
Y_{i} \sim \operatorname{Bernoulli}\left(\nu_{i}\right)
$$

with mean $\nu_{i}=\mathrm{E}\left(Y_{i}\right)$ and variance $\operatorname{var}\left(Y_{i}\right)=\nu_{i}\left(1-\nu_{i}\right)$. Let $\boldsymbol{x}_{i}=\left(1, x_{i 1}, \ldots, x_{i p}\right)^{\prime}$ denote the vector of covariates containing the value one and $p$ covariates at site $i$, and let $\boldsymbol{\beta}=\left(\beta_{0}, \beta_{1}, \ldots, \beta_{p}\right)^{\prime}$ denote a vector of regression coefficients including the intercept. We apply a probit link function to model the relation between the binary response $Y_{i} \in\{0,1\}$ and the covariates at site $i$ as

$$
\nu_{i}=\mathrm{E}\left(Y_{i}\right)=\Phi_{1}\left(\boldsymbol{x}_{i}^{\prime} \boldsymbol{\beta}\right) .
$$


Thus, parameterized by $\boldsymbol{\beta}$, the Bernoulli mass (or, density) function of $Y_{i}$ is

$$
f_{\mathrm{Bern}}\left(y_{i} ; \boldsymbol{\beta}\right)=y_{i} \nu_{i}+\left(1-y_{i}\right)\left(1-\nu_{i}\right)=y_{i} \Phi_{1}\left(\boldsymbol{x}_{i}^{\prime} \boldsymbol{\beta}\right)+\left(1-y_{i}\right)\left\{1-\Phi_{1}\left(\boldsymbol{x}_{i}^{\prime} \boldsymbol{\beta}\right)\right\}
$$

where the probability of one-value, $P\left(Y_{i}=1\right)=\Phi_{1}\left(\boldsymbol{x}_{i}^{\prime} \boldsymbol{\beta}\right)$, increases as $\boldsymbol{x}_{i}^{\prime} \boldsymbol{\beta}$ increases.

For the beta component, we let

$$
Y_{i} \sim \operatorname{Beta}\left(\mu_{i}, \phi\right)
$$

with mean $\mu_{i}=\mathrm{E}\left(Y_{i}\right) \in(0,1)$, variance $\operatorname{var}\left(Y_{i}\right)=\mu_{i}\left(1-\mu_{i}\right) / \phi \geq 0$, and a precision parameter $\phi>0$. We apply a logit link function to relate the proportional response $Y_{i} \in(0,1)$ to the covariates,

$$
\mu_{i}=\mathrm{E}\left(Y_{i}\right)=\left\{1+\exp \left(-\boldsymbol{x}_{i}^{\prime} \gamma\right)\right\}^{-1}
$$

where $\gamma=\left(\gamma_{0}, \gamma_{1}, \ldots, \gamma_{p}\right)^{\prime}$ is a vector of regression coefficients and $\mu_{i}$ increases as $\boldsymbol{x}_{i}^{\prime} \boldsymbol{\gamma}$ increases. Then, parameterized by $\gamma$ and $\phi$, the beta density function of $Y_{i}$ is

$$
f_{\text {Beta }}\left(y_{i} ; \gamma, \phi\right)=\frac{\Gamma(\phi)}{\Gamma\left(\mu_{i} \phi\right) \Gamma\left(\left(1-\mu_{i}\right) \phi\right)} y_{i}^{\mu_{i} \phi-1}\left(1-y_{i}\right)^{\left(1-\mu_{i}\right) \phi-1}
$$

where $\Gamma(t)=\int_{0}^{\infty} x^{t-1} e^{-x} d x$ is the gamma function. The beta distribution is flexible for modeling continuous data on the open interval $(0,1)$, since its probability density can take on a variety of shapes for different values of $\mu_{i}$ and $\phi$ including unimodal, uniform, "U", and "J" shaped distributions (Ferrari and Cribari-Neto, 2004; Ospina and Ferrari, 2010). As $\phi \rightarrow 0, Y_{i}$ tends to a Bernoulli random variable with success probability $P\left(Y_{i}=1\right)=\mu_{i}$, whereas as $\phi \rightarrow \infty, Y_{i}$ tends to a degenerate random variable with $P\left(Y_{i}=\mu_{i}\right)=1$ (Mielke, 1975).

From (2) and (5), the response $Y_{i}$ can relate to the covariates $\boldsymbol{x}_{i}$ via $\boldsymbol{\beta}$ and $\boldsymbol{\gamma}$ in the Bernoulli and the beta component, respectively. Now, to account for possible spatial dependence among the 
vector of responses at the $N$ sampling sites, $\boldsymbol{Y}=\left(Y_{1}, \ldots, Y_{N}\right)^{\prime}$, we introduce a vector of spatial latent variables $\boldsymbol{Y}^{*}=\left(Y_{1}^{*}, \ldots, Y_{N}^{*}\right)^{\prime}$, where at site $i$,

$$
Y_{i}^{*}=Z_{i}+\epsilon_{i}
$$

$Z_{i}$ is another spatial latent process, and $\epsilon_{i}$ is a measurement error, for $i=1, \ldots, N$. For $Z=$ $\left(Z_{1}, \ldots, Z_{N}\right)^{\prime}$, we let $\boldsymbol{Z} \sim N\left(\boldsymbol{X} \boldsymbol{\beta}, \boldsymbol{\Sigma}_{\boldsymbol{Z}}\right)$ follow a multivariate Gaussian distribution, where $\boldsymbol{X}=$ $\left(\boldsymbol{x}_{1}, \ldots, \boldsymbol{x}_{N}\right)^{\prime}$ denotes an $N \times(p+1)$ design matrix and $\boldsymbol{\Sigma}_{\boldsymbol{Z}}=\operatorname{var}(\boldsymbol{Z})$ is the covariance matrix of $\boldsymbol{Z}$. In particular, we assume an exponential covariance function $\operatorname{cov}\left(Z_{i}, Z_{i^{\prime}}\right)=\sigma^{2} \exp \left\{d_{i i^{\prime}} \log (\rho)\right\}$, or equivalently, $\operatorname{cov}\left(Z_{i}, Z_{i^{\prime}}\right)=\sigma^{2} \rho^{d_{i i^{\prime}}}$, where $d_{i i^{\prime}}$ is the Euclidean distance between sites $i$ and $i^{\prime}$, $\rho \in(0,1)$ is a spatial correlation parameter, and $\sigma^{2}=\operatorname{var}\left(Z_{i}\right)$ is a variance component. The measurement errors $\left\{\epsilon_{i}\right\}$ are assumed to be independently and identically distributed (iid) as $N\left(0, \tau^{2}\right)$ and are independent of $\boldsymbol{Z}$. Hence, $Y_{i}^{*} \sim N\left(\boldsymbol{x}_{i}^{\prime} \boldsymbol{\beta}, \sigma^{2}+\tau^{2}\right)$ and $\operatorname{cov}\left(Y_{i}^{*}, Y_{i^{\prime}}^{*}\right)=\sigma^{2} \rho^{d_{i i^{\prime}}}$ for $i \neq i^{\prime}$. We denote the covariance matrix of $\boldsymbol{Y}^{*}$ as $\boldsymbol{\Sigma}_{\boldsymbol{Y}^{*}}$ and thus, $\boldsymbol{Y}^{*} \sim N\left(\boldsymbol{X} \boldsymbol{\beta}, \boldsymbol{\Sigma}_{\boldsymbol{Y}^{*}}\right)$.

The Bernoulli component (1)-(2) can be re-expressed in the form of a threshold model,

$$
Y_{i}=\mathcal{I}\left(Y_{i}^{*}>c\right)
$$

where $Y_{i}^{*}$ is the spatial latent variable $(7), c \in \mathbb{R}$ is a threshold value, and $\mathcal{I}(\cdot)$ is the indicator function (see, e.g., Heagerty and Lele, 1998; De Oliveira, 2000). Thus, $\nu_{i}=\mathrm{E}\left(Y_{i}\right)=$ $\Phi_{1}\left(\left(\boldsymbol{x}_{i}^{\prime} \boldsymbol{\beta}-c\right) /\left(\sigma^{2}+\tau^{2}\right)\right)$. For parameter identification, we set $c=0$ and $\operatorname{var}\left(Y_{i}^{*}\right)=\sigma^{2}+\tau^{2}=1$.

The beta component (4)-(5) can be re-expressed as a transformation of the same latent variable $Y_{i}^{*}$,

$$
Y_{i}=F_{\text {Beta }}^{-1}\left(F_{\text {Gauss }}\left(Y_{i}^{*} ; \boldsymbol{\beta}\right) ; \boldsymbol{\gamma}, \phi\right)
$$

where $F_{\text {Beta }}^{-1}(\cdot ; \gamma, \phi)$ denotes the inverse CDF of a beta random variable parameterized by $\gamma$ and $\phi$, and $F_{\text {Gauss }}(\cdot ; \boldsymbol{\beta})$ denotes the Gaussian $\mathrm{CDF}$ of $Y_{i}^{*}$ parameterized by $\boldsymbol{\beta}$. It can be shown that 
$F_{\text {Gauss }}\left(Y_{i}^{*} ; \boldsymbol{\beta}\right)$ follows a standard uniform distribution and thus $F_{\text {Beta }}^{-1}\left(F_{\text {Gauss }}\left(Y_{i}^{*} ; \boldsymbol{\beta}\right) ; \boldsymbol{\gamma}, \phi\right)$ follows a beta distribution on $(0,1)$.

In summary, for site $i$, the spatial beta-Bernoulli mixture model with the Bernoulli component given in (8) and the beta component given in (9) can be rewritten as

$$
Y_{i}= \begin{cases}\mathcal{I}\left(Y_{i}^{*}>0\right) & \text { w.p. } \eta \\ F_{\text {Beta }}^{-1}\left(F_{\text {Gauss }}\left(Y_{i}^{*} ; \boldsymbol{\beta}\right) ; \boldsymbol{\gamma}, \phi\right) & \text { w.p. } 1-\eta\end{cases}
$$

where $\eta \in[0,1]$. When $\eta=1$, the spatial beta-Bernoulli mixture model 10$]$ reduces to a spatial probit model, whereas when $\eta=0$, it is a spatial beta model for spatial data on the open interval $(0,1)$ (Eskelson et al. 2011). Thus, the corresponding probability density function for $y_{i} \in[0,1]$ is

$$
f\left(y_{i} ; \eta, \boldsymbol{\beta}, \boldsymbol{\gamma}, \phi\right)= \begin{cases}\eta f_{\mathrm{Bern}}\left(y_{i} ; \boldsymbol{\beta}\right) & \text { if } y_{i}=\{0,1\} \\ (1-\eta) f_{\mathrm{Beta}}\left(y_{i} ; \boldsymbol{\gamma}, \phi\right) & \text { if } y_{i} \in(0,1)\end{cases}
$$

where $f\left(y_{i} ; \boldsymbol{\beta}, \boldsymbol{\gamma}, \phi\right)$ is parameterized by $\boldsymbol{\beta}$ when $y_{i}=0$ or 1 , and is parameterized by $\boldsymbol{\gamma}$ and $\phi$, but not $\boldsymbol{\beta}$, when $y_{i} \in(0,1)$. The beta density is due to $\partial P\left(Y_{i} \leq y_{i}\right) / \partial y_{i}=\partial P(0<$ $\left.Y_{i} \leq y_{i}\right) / \partial y_{i}=(1-\eta) \partial P\left(F_{\text {Gauss }}^{-1}\left(F_{\text {Beta }}\left(Y_{i} ; \boldsymbol{\gamma}, \phi\right) ; \boldsymbol{\beta}\right) \leq F_{\text {Gauss }}^{-1}\left(F_{\text {Beta }}\left(y_{i} ; \boldsymbol{\gamma}, \phi\right) ; \boldsymbol{\beta}\right)\right) / \partial y_{i}=(1-$ $\eta) \partial F_{\text {Beta }}\left(y_{i} ; \boldsymbol{\gamma}, \phi\right) / \partial y_{i}$. For a positive $\beta_{j}$, the $j$ th covariate is positively associated with the spatial latent variable, with other covariates held constant. Through thresholding $(8)$, the larger $x_{i j}$ corresponds to more probability mass at 1 . Further, for a positive $\gamma_{j}$, the $j$ th covariate is positively associated with the proportional responses, with other covariates held constant.

\section{Parameter Estimation and Statistical Inference}

\subsection{Composite Likelihood Estimation}

Let $\boldsymbol{\theta}=\left(\eta, \boldsymbol{\beta}^{\prime}, \sigma^{2}, \rho, \boldsymbol{\gamma}^{\prime}, \phi\right)^{\prime}$ denote the vector of model parameters, where $\boldsymbol{\beta}, \boldsymbol{\gamma} \in \mathbb{R}^{p+1}, \phi>$ 0 , and $\eta, \sigma^{2}, \rho \in(0,1)$. Without loss of generality, we assume that the first $m_{1}$ observations, 
$y_{1}, \ldots, y_{m_{1}}$, are proportions on $(0,1)$, the next $m_{2}$ observations, $y_{m_{1}+1}, \ldots, y_{m_{1}+m_{2}}$, are zeros, and the remainder observations, $y_{m_{1}+m_{2}+1}, \ldots, y_{N}$, are ones. The likelihood function of the parameter vector $\boldsymbol{\theta}=\left(\eta, \boldsymbol{\beta}, \sigma^{2}, \rho, \boldsymbol{\gamma}, \phi\right)^{\prime}$ is $\mathcal{L}(\boldsymbol{\theta})=(1-\eta)^{m_{1}} \eta^{N-m_{1}} \partial^{m_{1}} P\left(Y_{1} \leq y_{1}, \ldots, Y_{m_{1}} \leq y_{m_{1}}, Y_{m_{1}+1}=\right.$ $\left.0, \ldots, Y_{N}=1\right) / \partial y_{1} \cdots \partial y_{m_{1}}$, which can be re-expressed as

$\mathcal{L}(\boldsymbol{\theta})=(1-\eta)^{m_{1}} \eta^{N-m_{1}} \frac{\partial^{m_{1}} P\left(Y_{1}^{*} \leq F_{\text {Gauss }}^{-1}\left(F_{\text {Beta }}\left(y_{1} ; \boldsymbol{\gamma}, \phi\right) ; \boldsymbol{\beta}\right), \ldots, Y_{m_{1}+1}^{*} \leq 0, \ldots, Y_{N}^{*}>0\right)}{\partial y_{1} \cdots \partial y_{m_{1}}}$

The likelihood function (12) involves the operations of $N \times N$ matrices and multiple integrals and thus, its maximization becomes infeasible especially when $N$ is large and when the amount of zero/one values is large.

Taking the approach of composite likelihood, we define a pairwise likelihood function for $\boldsymbol{\theta}$ as

$$
\mathcal{L}_{\text {pair }}(\boldsymbol{\theta})=\prod_{i=1}^{N-1} \prod_{i^{\prime}=i+1}^{N} f\left(y_{i}, y_{i^{\prime}} ; \boldsymbol{\theta}\right)
$$

In particular, for a pair of sites $\left(i, i^{\prime}\right)$, the pair of responses $\left(Y_{i}, Y_{i^{\prime}}\right)$ have three possible scenarios: both of them are integers ( 0 or 1$)$, one is an integer and the other is a proportion, or both are proportions. In the first scenario, the bivariate densities involve $\eta, \boldsymbol{\beta}, \sigma^{2}$, and $\rho$, but not $\boldsymbol{\gamma}$ and $\phi$ as 
follows:

$$
\begin{aligned}
f_{\mathrm{Bern} 2}\left(0,0 ; \eta, \boldsymbol{\beta}, \sigma^{2}, \rho\right) & =P\left(Y_{i}=0, Y_{i^{\prime}}=0\right)=\eta^{2} P\left(Y_{i}^{*} \leq 0, Y_{i^{\prime}}^{*} \leq 0\right) \\
& =\eta^{2} \Phi_{2}\left(-\boldsymbol{x}_{i}^{\prime} \boldsymbol{\beta},-\boldsymbol{x}_{i^{\prime}}^{\prime} \boldsymbol{\beta} ; \tilde{\rho}_{i i^{\prime}}\right), \\
f_{\mathrm{Bern} 2}\left(0,1 ; \eta, \boldsymbol{\beta}, \sigma^{2}, \rho\right) & =P\left(Y_{i}=0, Y_{i^{\prime}}=1\right)=\eta^{2} P\left(Y_{i}^{*} \leq 0, Y_{i^{\prime}}^{*}>0\right) \\
& =\eta^{2}\left\{\Phi_{1}\left(-\boldsymbol{x}_{i}^{\prime} \boldsymbol{\beta}\right)-\Phi_{2}\left(-\boldsymbol{x}_{i}^{\prime} \boldsymbol{\beta},-\boldsymbol{x}_{i^{\prime}}^{\prime} \boldsymbol{\beta} ; \tilde{\rho}_{i i^{\prime}}\right)\right\} \\
f_{\mathrm{Bern} 2}\left(1,0 ; \eta, \boldsymbol{\beta}, \sigma^{2}, \rho\right) & =P\left(Y_{i}=1, Y_{i^{\prime}}=0\right)=\eta^{2} P\left(Y_{i}^{*}>0, Y_{i^{\prime}}^{*} \leq 0\right) \\
& =\eta^{2}\left\{\Phi_{1}\left(-\boldsymbol{x}_{i^{\prime}}^{\prime} \boldsymbol{\beta}\right)-\Phi_{2}\left(-\boldsymbol{x}_{i}^{\prime} \boldsymbol{\beta},-\boldsymbol{x}_{i^{\prime}}^{\prime} \boldsymbol{\beta} ; \tilde{\rho}_{i i^{\prime}}\right)\right\} \\
f_{\mathrm{Bern} 2}\left(1,1 ; \eta, \boldsymbol{\beta}, \sigma^{2}, \rho\right) & =P\left(Y_{i}=1, Y_{i^{\prime}}=1\right)=\eta^{2} P\left(Y_{i}^{*}>0, Y_{i^{\prime}}^{*}>0\right) \\
& =\eta^{2} \Phi_{2}\left(\boldsymbol{x}_{i}^{\prime} \boldsymbol{\beta}, \boldsymbol{x}_{i^{\prime}}^{\prime} \boldsymbol{\beta} ; \tilde{\rho}_{i i^{\prime}}\right),
\end{aligned}
$$

where $\Phi_{2}\left(\nu_{1}, \nu_{2} ; \tilde{\rho}_{i i^{\prime}}\right)=(2 \pi)^{-1}\left(1-\tilde{\rho}_{i i^{\prime}}^{2}\right)^{-1 / 2} \int_{-\infty}^{\nu_{1}} \int_{-\infty}^{\nu_{2}} \exp \left\{\left(2-2 \tilde{\rho}_{i i^{\prime}}^{2}\right)^{-1}\left(t_{1}^{2}+t_{2}^{2}-2 \tilde{\rho}_{i i^{\prime}} t_{1} t_{2}\right)\right\} d t_{1} d t_{2}$ is the CDF of the standard bivariate Gaussian distribution with means 0, variances 1, and correlation $\tilde{\rho}_{i i^{\prime}}=\sigma^{2} \rho^{d_{i i^{\prime}}}$.

In the second scenario, one response is an integer and the other is a proportion. The bivariate density can be derived in a straightforward manner and involve all the parameters $\boldsymbol{\theta}$. For example, suppose $y_{i}=0$ and $y_{i^{\prime}} \in(0,1)$, since

$$
\begin{aligned}
P\left(Y_{i}=0,0<Y_{i^{\prime}} \leq y_{i^{\prime}}\right) & =\eta(1-\eta) P\left(Y_{i}^{*} \leq 0, h\left(Y_{i^{\prime}} ; \boldsymbol{\beta}, \boldsymbol{\gamma}, \phi\right) \leq h\left(y_{i^{\prime}} ; \boldsymbol{\beta}, \boldsymbol{\gamma}, \phi\right)\right) \\
& =\eta(1-\eta) P\left(Y_{i}^{*}-\boldsymbol{x}_{i}^{\prime} \boldsymbol{\beta} \leq-\boldsymbol{x}_{i}^{\prime} \boldsymbol{\beta}, Y_{i^{\prime}}^{*}-\boldsymbol{x}_{i^{\prime}}^{\prime} \boldsymbol{\beta} \leq h\left(y_{i^{\prime}} ; \boldsymbol{\beta}, \boldsymbol{\gamma}, \phi\right)-\boldsymbol{x}_{i^{\prime}}^{\prime} \boldsymbol{\beta}\right) \\
& =\eta(1-\eta) P\left(Y_{i}^{*}-\boldsymbol{x}_{i}^{\prime} \boldsymbol{\beta} \leq-\boldsymbol{x}_{i}^{\prime} \boldsymbol{\beta}, Y_{i^{\prime}}^{*}-\boldsymbol{x}_{i^{\prime}}^{\prime} \boldsymbol{\beta} \leq u\left(y_{i^{\prime}} ; \boldsymbol{\gamma}, \phi\right)\right) \\
& =\eta(1-\eta) \Phi_{2}\left(-\boldsymbol{x}_{i}^{\prime} \boldsymbol{\beta}, u\left(y_{i^{\prime}} ; \boldsymbol{\gamma}, \phi\right) ; \tilde{\rho}_{i i^{\prime}}\right)
\end{aligned}
$$

where $h(\cdot ; \boldsymbol{\beta}, \boldsymbol{\gamma}, \phi)=F_{\text {Gauss }}^{-1}\left(F_{\text {Beta }}(\cdot, \boldsymbol{\gamma}, \phi), \boldsymbol{\beta}\right)$ and $u\left(y_{i^{\prime}} ; \boldsymbol{\gamma}, \phi\right)=h\left(y_{i^{\prime}} ; \boldsymbol{\beta}, \boldsymbol{\gamma}, \phi\right)-\boldsymbol{x}_{i^{\prime}}^{\prime} \boldsymbol{\beta}=\Phi_{1}^{-1}\left(F_{\text {Beta }}\left(y_{i^{\prime}} ; \boldsymbol{\gamma}, \phi\right)\right)$ 
Then, we have

$$
\begin{aligned}
f_{\text {Bern,Beta }}\left(0, y_{i^{\prime}} ; \boldsymbol{\theta}\right)= & \partial P\left(Y_{i}=0, Y_{i^{\prime}} \leq y_{i^{\prime}}\right) / \partial y_{i^{\prime}}=\partial P\left(Y_{i}=0,0<Y_{i^{\prime}} \leq y_{i^{\prime}}\right) / \partial y_{i^{\prime}} \\
= & \eta(1-\eta) \partial \Phi_{2}\left(-\boldsymbol{x}_{i}^{\prime} \boldsymbol{\beta}, u\left(y_{i^{\prime}} ; \boldsymbol{\gamma}, \phi\right) ; \tilde{\rho}_{i i^{\prime}}\right) / \partial y_{i^{\prime}} \\
= & \eta(1-\eta) \Phi_{1}\left(\left(-\boldsymbol{x}_{i}^{\prime} \boldsymbol{\beta}-\tilde{\rho}_{i i^{\prime}} u\left(y_{i^{\prime}} ; \boldsymbol{\gamma}, \phi\right)\right)\left(1-\tilde{\rho}_{i i^{\prime}}^{2}\right)^{-1 / 2}\right) \\
= & \eta(1-\eta) \Phi_{1}\left(\left(-\boldsymbol{x}_{i}^{\prime} \boldsymbol{\beta}-\tilde{\rho}_{i i^{\prime}} u\left(y_{i^{\prime}} ; \boldsymbol{\gamma}, \phi\right)\right)\left(1-\tilde{\rho}_{i i^{\prime}}^{2}\right)^{-1 / 2}\right) \\
& \times \phi_{1}\left(u\left(y_{i^{\prime}} ; \boldsymbol{\gamma}, \phi\right)\right) \partial u\left(y_{i^{\prime}} ; \boldsymbol{\gamma}, \phi\right) / \partial y_{i^{\prime}} \\
= & \eta(1-\eta) \Phi_{1}\left(\left(-\boldsymbol{x}_{i}^{\prime} \boldsymbol{\beta}-\tilde{\rho}_{i i^{\prime}} u\left(y_{i^{\prime}} ; \boldsymbol{\gamma}, \phi\right)\right)\left(1-\tilde{\rho}_{i i^{\prime}}^{2}\right)^{-1 / 2}\right) \\
& \times \phi_{1}\left(u\left(y_{i^{\prime}} ; \boldsymbol{\gamma}, \phi\right)\right) f_{\text {Beta }}\left(y_{i^{\prime}} ; \boldsymbol{\gamma}, \phi\right) / \phi \phi_{1}\left(u\left(y_{i^{\prime}} ; \boldsymbol{\gamma}, \phi\right)\right) \\
= & \eta(1-\eta) \Phi_{1}\left(\left(-\boldsymbol{x}_{i}^{\prime} \boldsymbol{\beta}-\tilde{\rho}_{i i^{\prime}} u\left(y_{i^{\prime}} ; \boldsymbol{\gamma}, \phi\right)\right)\left(1-\tilde{\rho}_{i i^{\prime}}^{2}\right)^{-1 / 2}\right) f_{\text {Beta }}\left(y_{i^{\prime}} ; \boldsymbol{\gamma}, \phi\right) .
\end{aligned}
$$

Under the independence assumption (i.e., $\rho=0$ ), the bivariate density $(15)$ is simplified to the product of Bernoulli and beta densities $f_{\text {Bern,Beta }}\left(0, y_{i^{\prime}} ; \boldsymbol{\theta}\right)=\eta(1-\eta) f_{\text {Beta }}\left(y_{i^{\prime}} ; \boldsymbol{\gamma}, \phi\right) \Phi_{1}\left(-\boldsymbol{x}_{i}^{\prime} \boldsymbol{\beta}\right)$. By analogous arguments,

$$
\begin{aligned}
f_{\text {Bern,Beta }}\left(1, y_{i^{\prime}} ; \boldsymbol{\theta}\right) & =\eta(1-\eta) f_{\text {Beta }}\left(y_{i^{\prime}} ; \boldsymbol{\gamma}, \phi\right) \Phi_{1}\left(\left(\boldsymbol{x}_{i}^{\prime} \boldsymbol{\beta}+\tilde{\rho}_{i i^{\prime}} u\left(y_{i^{\prime}} ; \boldsymbol{\gamma}, \phi\right)\right)\left(1-\tilde{\rho}_{i i^{\prime}}^{2}\right)^{-1 / 2}\right) \\
f_{\text {Beta,Bern }}\left(y_{i}, 0 ; \boldsymbol{\theta}\right) & =\eta(1-\eta) f_{\text {Beta }}\left(y_{i} ; \boldsymbol{\gamma}, \phi\right) \Phi_{1}\left(\left(-\boldsymbol{x}_{i^{\prime}}^{\prime} \boldsymbol{\beta}-\tilde{\rho}_{i i^{\prime}} u\left(y_{i} ; \boldsymbol{\gamma}, \phi\right)\right)\left(1-\tilde{\rho}_{i i^{\prime}}^{2}\right)^{-1 / 2}\right) \\
f_{\text {Beta,Bern }}\left(y_{i}, 1 ; \boldsymbol{\theta}\right) & =\eta(1-\eta) f_{\text {Beta }}\left(y_{i} ; \boldsymbol{\gamma}, \phi\right) \Phi_{1}\left(\left(\boldsymbol{x}_{i^{\prime}}^{\prime} \boldsymbol{\beta}+\tilde{\rho}_{i i^{\prime}} u\left(y_{i} ; \boldsymbol{\gamma}, \phi\right)\right)\left(1-\tilde{\rho}_{i i^{\prime}}^{2}\right)^{-1 / 2}\right) .
\end{aligned}
$$

In the third scenario, both responses are proportions. The bivariate density involves $\eta, \gamma, \phi$, and 
the spatial dependence parameters $\sigma^{2}$ and $\rho$, but not $\boldsymbol{\beta}$,

$$
\begin{aligned}
f_{\text {Beta2 }}\left(y_{i}, y_{i^{\prime}} ; \eta, \gamma, \phi, \sigma^{2}, \rho\right)= & \frac{\partial^{2} P\left(Y_{i} \leq y_{i}, Y_{i^{\prime}} \leq y_{i^{\prime}}\right)}{\partial y_{i} \partial y_{i^{\prime}}}=(1-\eta)^{2} \frac{\partial^{2} \Phi_{2}\left(u_{i}, u_{i^{\prime}} ; \tilde{\rho}_{i i^{\prime}}\right)}{\partial y_{i} \partial y_{i^{\prime}}} \\
= & (1-\eta)^{2} f_{\text {Beta }}\left(y_{i} ; \boldsymbol{\gamma}, \phi\right) f_{\text {Beta }}\left(y_{i^{\prime}} ; \boldsymbol{\gamma}, \phi\right) \\
& \times \phi_{2}\left(u\left(y_{i} ; \boldsymbol{\gamma}, \phi\right), u\left(y_{i^{\prime}} ; \boldsymbol{\gamma}, \phi\right) ; \tilde{\rho}_{i i^{\prime}}\right) /\left\{\phi_{1}\left(u\left(y_{i} ; \boldsymbol{\gamma}, \phi\right)\right) \phi_{1}\left(u\left(y_{i^{\prime}} ; \boldsymbol{\gamma}, \phi\right)\right)\right\}
\end{aligned}
$$

Again, under the independence assumption, the bivariate density $(16)$ is reduced to $f_{\mathrm{Beta2}}\left(y_{i}, y_{i^{\prime}} ; \eta, \gamma, \phi\right)=$ $(1-\eta)^{2} f_{\text {Beta }}\left(y_{i} ; \gamma, \phi\right) f_{\text {Beta }}\left(y_{i^{\prime}} ; \gamma, \phi\right)$.

In summary, the bivariate density of the paired response $\left(Y_{i}, Y_{i^{\prime}}\right)$ is

$$
f\left(y_{i}, y_{i^{\prime}} ; \boldsymbol{\theta}\right)= \begin{cases}f_{\mathrm{Bern} 2}\left(y_{i}, y_{i^{\prime}} ; \eta, \boldsymbol{\beta}, \sigma^{2}, \rho\right) & \text { if } y_{i}, y_{i^{\prime}} \in\{0,1\} \\ f_{\mathrm{Bern}, \mathrm{Beta}}\left(y_{i}, y_{i^{\prime}} ; \boldsymbol{\theta}\right) & \text { if } y_{i} \in\{0,1\}, y_{i^{\prime}} \in(0,1) \\ f_{\mathrm{Beta}, \mathrm{Bern}}\left(y_{i}, y_{i^{\prime}} ; \boldsymbol{\theta}\right) & \text { if } y_{i} \in(0,1), y_{i^{\prime}} \in\{0,1\} \\ f_{\mathrm{Beta2} 2}\left(y_{i}, y_{i^{\prime}} ; \eta, \gamma, \phi\right) & \text { if } y_{i}, y_{i^{\prime}} \in(0,1) .\end{cases}
$$

Since the composite likelihood function 13 contains $N(N-1) / 2$ pairs of bivariate densities, the computation can still be quite time consuming for a large sample size $N$. Thus, we adopt a 0-1 weighting mechanism and define a weighted composite log-likelihood function as

$$
c \ell(\boldsymbol{\theta} ; \boldsymbol{y})=\sum_{i<i^{\prime}: d_{i i^{\prime}} \leq r} \log \left\{f\left(y_{i}, y_{i^{\prime}} ; \boldsymbol{\theta}\right)\right\}
$$

where $r$ is a pre-specified radius. That is, any pair farther than $r$ units apart is excluded via a zero weight (see, e.g., Heagerty and Lele 1998). With an exponential covariance function, the spatial correlation declines relatively fast as the distance increases between two sites and thus, this weighting scheme effectively excludes the distant pairs that have negligible correlation.

The maximum composite likelihood estimator (MCLE), denoted as $\hat{\boldsymbol{\theta}}=\left(\hat{\eta}, \hat{\boldsymbol{\beta}}^{\prime}, \hat{\sigma}^{2}, \hat{\rho}, \hat{\boldsymbol{\gamma}}^{\prime}, \hat{\phi}\right)^{\prime}$, 
maximizes $c \ell(\boldsymbol{\theta} ; \boldsymbol{y})$ and can be obtained by solving the score equations

$$
\boldsymbol{S}_{N}(\boldsymbol{\theta})=\frac{\partial}{\partial \boldsymbol{\theta}} c \ell(\boldsymbol{\theta} ; \boldsymbol{y})=\mathbf{0}
$$

using a quasi-Newton algorithm for bounded parameter space (Byrd et al., 1995). Because each individual component in (18) is a bivariate density, the resulting score equations (19) obtained from the derivative of the composite log-likelihood are unbiased (Varin et al., 2011). The consistency and asymptotic normality of $\hat{\boldsymbol{\theta}}$ can be derived under suitable regularity conditions using arguments similar to Guyon (1995).

\subsection{Statistical Inference}

The variance of $\hat{\boldsymbol{\theta}}$ can be obtained by computing the inverse of the Godambe information matrix

$$
\boldsymbol{G}_{N}^{-1}(\boldsymbol{\theta})=\boldsymbol{H}_{N}^{-1}(\boldsymbol{\theta}) \boldsymbol{J}_{N}(\boldsymbol{\theta}) \boldsymbol{H}_{N}^{-1}(\boldsymbol{\theta})
$$

where $\boldsymbol{H}_{N}(\boldsymbol{\theta})=-E\left(\frac{\partial}{\partial \boldsymbol{\theta}^{\prime}} \boldsymbol{S}_{N}(\boldsymbol{\theta})\right)$ is the sensitivity matrix and $\boldsymbol{J}_{N}(\boldsymbol{\theta})=\operatorname{var}\left(\boldsymbol{S}_{N}(\boldsymbol{\theta})\right)$ is the variability matrix (Godambe, 1960). In practice, we approximate $\boldsymbol{H}_{N}(\boldsymbol{\theta})$ by a numerical Hessian matrix, and $\boldsymbol{J}_{N}(\boldsymbol{\theta})$ by a sample covariance matrix of the score functions, $\boldsymbol{S}_{N}\left(\hat{\boldsymbol{\theta}} ; \boldsymbol{y}_{1}\right), \ldots, \boldsymbol{S}_{N}\left(\hat{\boldsymbol{\theta}} ; \boldsymbol{y}_{M}\right)$, denoted as $\hat{\boldsymbol{J}}_{N}(\hat{\boldsymbol{\theta}})$, where $\boldsymbol{y}_{m}=\left(y_{m 1}, \ldots, y_{m N}\right)^{\prime}$ for $m=1, \ldots, M$ are independent draws from the fitted model (Apanasovich et al., 2008, Varin et al., 2011). Since closed-form score functions are not straightforward to derive, we obtain the sample covariance matrix based on the numerical score functions $\hat{S}_{N}\left(\theta_{j} ; \boldsymbol{y}\right)=\left\{c \ell\left(\theta_{j}+\delta ; \boldsymbol{y}\right)-c \ell\left(\theta_{j}-\delta ; \boldsymbol{y}\right)\right\} /(2 \delta)$, for $j=1, \ldots, 2 p+6$, where $\delta>0$ is a small value and $p+1$ is the number of regression coefficients for $\boldsymbol{\beta}$ or $\boldsymbol{\gamma}$. For $m=1, \ldots, M$, we draw the latent variable $\boldsymbol{Y}_{m}^{*} \sim N\left(\boldsymbol{X} \hat{\boldsymbol{\beta}}, \hat{\boldsymbol{\Sigma}}_{\boldsymbol{Y}^{*}}\right)$ and then draw the response $\boldsymbol{y}_{m}$ from 10 evaluated at the parameter estimates. This procedure can be further expedited via parallel computing.

For model comparison, we consider a composite likelihood information criterion (CLIC) (Varin and Vidoni, 2005), which is a generalization of Akaike's information criterion (AIC) for model 
assessment (Akaike, 1973). Specifically, for (18), the CLIC is given by

$$
\operatorname{CLIC}(\hat{\boldsymbol{\theta}})=-2 c l(\hat{\boldsymbol{\theta}})+2 \operatorname{tr}\left(\hat{\boldsymbol{J}}_{N}(\hat{\boldsymbol{\theta}}) \hat{\boldsymbol{H}}_{N}^{-1}(\hat{\boldsymbol{\theta}})\right)
$$

When the composite likelihood is the true likelihood, (21) is the same as AIC, because $\boldsymbol{J}_{N}(\boldsymbol{\theta})=$ $\boldsymbol{H}_{N}(\boldsymbol{\theta})$ and the trace corresponds to the number of parameters in the model.

\section{Spatial Generalized Tobit Model}

In Sections 2 and 3, spatial proportional data with zero/one values are modeled by mixing a beta distribution and a Bernoulli distribution. Alternatively, we consider imposing a functional transformation on the latent variable. In particular, we propose a spatial generalized Tobit model, which extends a spatial Tobit model by applying an inverse beta CDF transformation to the latent variable. That is, let

$$
Y_{i}= \begin{cases}1 & \text { if } Y_{i}^{*}>\alpha \\ F_{\text {Beta }}^{-1}\left(Y_{i}^{*} / \alpha ; \gamma, \phi\right) & \text { if } 0<Y_{i}^{*} \leq \alpha \\ 0 & \text { if } Y_{i}^{*} \leq 0,\end{cases}
$$

where $Y_{i}^{*}$ is a spatial latent variable given in (7). Recall that $\boldsymbol{Y}^{*}=\left(Y_{1}^{*}, \ldots, Y_{N}^{*}\right)^{\prime}, \boldsymbol{Y}^{*} \sim$ $N\left(\boldsymbol{X} \boldsymbol{\beta}, \boldsymbol{\Sigma}_{\boldsymbol{Y}^{*}}\right)$, and the $(i, j)$ th term of the covariance matrix $\boldsymbol{\Sigma}_{\boldsymbol{Y}^{*}}$ is $\sigma^{2} \rho^{d_{i j}}$ for $i, j=1, \ldots, N$. Further, we consider a logit link function for the mean of the beta distribution $\mu_{i}=\mathrm{E}\left(Y_{i}\right)=$ $\left\{1+\exp \left(-\boldsymbol{x}_{i}^{\prime} \boldsymbol{\gamma}\right)\right\}^{-1}$ for $Y_{i} \in(0,1)$, and $\boldsymbol{\gamma}=\left(\gamma_{0}, \gamma_{1}, \ldots, \gamma_{p}\right)^{\prime}$ is a vector of regression coefficients.

In (22), the parameter $\alpha \geq 0$ is a threshold value and controls the probability of $Y_{i}=1$. The point mass at one is larger as $\alpha$ decreases. For $\alpha=0$, model (22) is reduced to a spatial probit model for binary responses, while for $\alpha>0$, the latent variable $Y_{i}^{*}$ between 0 and $\alpha$ is transformed to be between 0 and 1 . For $\operatorname{Beta}(0.5,2)$ with $\mu=0.5$ and $\phi=2$, the beta distribution is the 
standard uniform distribution and thus, (22) is reduced to a spatial Tobit model. The inverse beta CDF transformation $F_{\text {Beta }}^{-1}\left(Y_{i}^{*} / \alpha ; \gamma, \phi\right)$ is a new idea aimed to overcome the unimodality implied by the Gaussian latent variable in the existing spatial Tobit model.

The vector of model parameters is $\boldsymbol{\theta}=\left(\alpha, \boldsymbol{\beta}^{\prime}, \sigma^{2}, \rho, \boldsymbol{\gamma}^{\prime}, \phi\right)^{\prime}$, where $\boldsymbol{\beta}, \boldsymbol{\gamma} \in \mathbb{R}^{p+1}, \phi>0, \alpha \geq 0$, and $\sigma^{2}, \rho \in(0,1)$. The corresponding probability density function at site $i$ is given by

$$
f\left(y_{i} ; \boldsymbol{\theta}\right)= \begin{cases}\Phi_{1}\left(\boldsymbol{x}_{i}^{\prime} \boldsymbol{\beta}-\alpha\right) & \text { if } y_{i}=1 \\ \alpha f_{\mathrm{Beta}}\left(y_{i} ; \boldsymbol{\gamma}, \phi\right) \phi_{1}\left(\alpha F_{\mathrm{Beta}}\left(y_{i} ; \boldsymbol{\gamma}, \phi\right)-\boldsymbol{x}_{i}^{\prime} \boldsymbol{\beta}\right) & \text { if } y_{i} \in(0,1) \\ \Phi_{1}\left(-\boldsymbol{x}_{i}^{\prime} \boldsymbol{\beta}\right) & \text { if } y_{i}=0,\end{cases}
$$

where $\phi_{1}(x)=(2 \pi)^{-1 / 2} \exp \left(x^{2} / 2\right)$ is the density of the standard Gaussian distribution. The density for $y_{i} \in(0,1)$ is due to $\partial P\left(Y_{i} \leq y_{i}\right) / \partial y_{i}=\partial P\left(\alpha F_{\text {Beta }}\left(Y_{i} ; \boldsymbol{\gamma}, \phi\right) \leq \alpha F_{\text {Beta }}\left(y_{i} ; \gamma, \phi\right)\right) / \partial y_{i}=$ $\partial P\left(\alpha F_{\text {Beta }}\left(Y_{i} ; \boldsymbol{\gamma}, \phi\right)-\boldsymbol{x}_{i}^{\prime} \boldsymbol{\beta} \leq \alpha F_{\text {Beta }}\left(y_{i} ; \boldsymbol{\gamma}, \phi\right)-\boldsymbol{x}_{i}^{\prime} \boldsymbol{\beta}\right) / \partial y_{i}=\partial \Phi_{1}\left(\alpha F_{\text {Beta }}\left(y_{i} ; \boldsymbol{\gamma}, \phi\right)-\boldsymbol{x}_{i}^{\prime} \boldsymbol{\beta}\right) / \partial y_{i}$. Unlike the density (11) under the spatial beta-Bernoulli mixture model, the regression coefficients $\boldsymbol{\beta}$ under the spatial generalized Tobit model appear in both the distribution of zero/one values and the proportions. For a positive $\beta_{j}$, there is more probability mass at 1 and less at 0 as $x_{i j}$ increases with other covariates hold constant, while the effect of $x_{i j}$ varies for $y_{i} \in(0,1)$.

The maximum likelihood estimation for model (22) is again not feasible for relatively large sample sizes and thus, we take a composite likelihood approach. Similar to Section 2, for a pair of sites $\left(i, i^{\prime}\right)$, the pair of responses $\left(Y_{i}, Y_{i^{\prime}}\right)$ have three possible scenarios. When both of them are integers ( 0 or 1$)$, the bivariate density is the same as the spatial Tobit model. That is, parameterized by $\alpha, \boldsymbol{\beta}, \sigma^{2}, \rho$ but not $\gamma, \phi$,

$$
\begin{aligned}
& g_{\mathrm{Bern} 2}\left(0,0 ; \alpha, \boldsymbol{\beta}, \sigma^{2}, \rho\right)=P\left(Y_{i}=0, Y_{i^{\prime}}=0\right)=\Phi_{2}\left(-\boldsymbol{x}_{i}^{\prime} \boldsymbol{\beta},-\boldsymbol{x}_{i^{\prime}}^{\prime} \boldsymbol{\beta} ; \tilde{\rho}_{i i^{\prime}}\right) \\
& g_{\mathrm{Bern} 2}\left(0,1 ; \alpha, \boldsymbol{\beta}, \sigma^{2}, \rho\right)=P\left(Y_{i}=0, Y_{i^{\prime}}=1\right)=\Phi_{1}\left(-\boldsymbol{x}_{i}^{\prime} \boldsymbol{\beta}\right)-\Phi_{2}\left(-\boldsymbol{x}_{i}^{\prime} \boldsymbol{\beta}, \alpha-\boldsymbol{x}_{i^{\prime}}^{\prime} \boldsymbol{\beta} ; \tilde{\rho}_{i i^{\prime}}\right) \\
& g_{\mathrm{Bern} 2}\left(1,0 ; \alpha, \boldsymbol{\beta}, \sigma^{2}, \rho\right)=P\left(Y_{i}=1, Y_{i^{\prime}}=0\right)=\Phi_{1}\left(-\boldsymbol{x}_{i^{\prime}}^{\prime} \boldsymbol{\beta}\right)-\Phi_{2}\left(\alpha-\boldsymbol{x}_{i}^{\prime} \boldsymbol{\beta},-\boldsymbol{x}_{i^{\prime}}^{\prime} \boldsymbol{\beta} ; \tilde{\rho}_{i i^{\prime}}\right) \\
& g_{\mathrm{Bern} 2}\left(1,1 ; \alpha, \boldsymbol{\beta}, \sigma^{2}, \rho\right)=P\left(Y_{i}=1, Y_{i^{\prime}}=1\right)=\Phi_{2}\left(\boldsymbol{x}_{i}^{\prime} \boldsymbol{\beta}-\alpha, \boldsymbol{x}_{i^{\prime}}^{\prime} \boldsymbol{\beta}-\alpha ; \tilde{\rho}_{i i^{\prime}}\right),
\end{aligned}
$$


where $\tilde{\rho}_{i i^{\prime}}=\sigma^{2} \rho^{d_{i i^{\prime}}}$.

When one response is binary and the other is a proportion (e.g., $y_{i}=0$ and $y_{i^{\prime}} \in(0,1)$ ), the bivariate density is parameterized by $\boldsymbol{\theta}$ as,

$$
\begin{aligned}
& g_{\text {Bern,Beta }}\left(0, y_{i^{\prime}} ; \boldsymbol{\theta}\right)=\partial P\left(Y_{i}=0, Y_{i^{\prime}} \leq y_{i^{\prime}}\right) / \partial y_{i^{\prime}} \\
= & \alpha \phi_{1}\left(v\left(y_{i} ; \boldsymbol{\beta}, \boldsymbol{\gamma}, \phi\right)\right) f_{\text {Beta }}\left(y_{i^{\prime}} ; \boldsymbol{\gamma}, \phi\right) \Phi_{1}\left(\left(\boldsymbol{x}_{i^{\prime}}^{\prime} \boldsymbol{\beta}-\alpha+\tilde{\rho}_{i i^{\prime}} v\left(y_{i} ; \boldsymbol{\beta}, \boldsymbol{\gamma}, \phi\right)\right)\left(1-\tilde{\rho}_{i i^{\prime}}^{2}\right)^{-1 / 2}\right),
\end{aligned}
$$

where $v(\cdot ; \boldsymbol{\beta}, \boldsymbol{\gamma}, \phi)=\alpha F_{\text {Beta }}\left(y_{i} ; \boldsymbol{\gamma}, \phi\right)-\boldsymbol{x}_{i}^{\prime} \boldsymbol{\beta}$ and $f_{\text {Beta }}\left(y_{i} ; \boldsymbol{\gamma}, \phi\right)$ is a beta probability density function. Unlike $u(\cdot ; \gamma, \phi)$ in the bivariate density $(15)$ of the spatial beta-Bernoulli mixture model, $v(\cdot ; \boldsymbol{\beta}, \boldsymbol{\gamma}, \phi)$ here cannot be further simplified and thus contains both $\boldsymbol{\beta}$ and $\boldsymbol{\gamma}$.

When both responses are proportions, the bivariate density is parameterized by $\boldsymbol{\theta}$,

$$
\begin{aligned}
g_{\text {Beta2 }}\left(y_{i}, y_{i^{\prime}} ; \boldsymbol{\theta}\right) & =\partial P\left(Y_{i} \leq y_{i^{\prime}}, Y_{i^{\prime}} \leq y_{i^{\prime}}\right) / \partial y_{i} \partial y_{i^{\prime}} \\
& =\alpha^{2} \phi_{2}\left(v\left(y_{i} ; \boldsymbol{\beta}, \boldsymbol{\gamma}, \phi\right), v\left(y_{i^{\prime}} ; \boldsymbol{\beta}, \boldsymbol{\gamma}, \phi\right) ; \tilde{\rho}_{i i^{\prime}}\right) f_{\text {Beta }}\left(y_{i} ; \boldsymbol{\gamma}, \phi\right) f_{\text {Beta }}\left(y_{i^{\prime}} ; \boldsymbol{\gamma}, \phi\right)
\end{aligned}
$$

where recall $v(\cdot ; \boldsymbol{\beta}, \boldsymbol{\gamma}, \phi)=\alpha F_{\text {Beta }}(\cdot ; \boldsymbol{\gamma}, \phi)-\boldsymbol{x}_{i}^{\prime} \boldsymbol{\beta}$. In summary, the bivariate density is

$$
g\left(y_{i}, y_{i^{\prime}} ; \boldsymbol{\theta}\right)= \begin{cases}g_{\text {Bern2 }}\left(y_{i}, y_{i^{\prime}} ; \alpha, \boldsymbol{\beta}, \sigma^{2}, \rho\right) & \text { if } y_{i}, y_{i^{\prime}} \in\{0,1\} \\ g_{\text {Bern,Beta }}\left(y_{i}, y_{i^{\prime}} ; \boldsymbol{\theta}\right) & \text { if } y_{i} \in\{0,1\}, y_{i^{\prime}} \in(0,1) \\ g_{\text {Beta,Bern }}\left(y_{i}, y_{i^{\prime}} ; \boldsymbol{\theta}\right) & \text { if } y_{i} \in(0,1), y_{i^{\prime}} \in\{0,1\} \\ g_{\text {Beta2 }}\left(y_{i}, y_{i^{\prime}} ; \boldsymbol{\theta}\right) & \text { if } y_{i}, y_{i^{\prime}} \in(0,1) .\end{cases}
$$

The computation of the MCLE, their standard errors, and CLIC is analogous to the spatial betaBernoulli mixture model in Section 3.2. The consistency and asymptotic normality of the maximum composite likelihood estimates $\hat{\boldsymbol{\theta}}$ can also be derived under suitable regularity conditions. 


\section{Simulation Study}

To examine the finite-sample properties of the methods developed in Section $2-4$, we conducted a simulation study on an $L \times L$ square lattice. First, data were generated under the spatial betaBernoulli mixture model (10), where $L=30$ or 40 , corresponding to sample size $N=900$ or 1600 . We let the mixture probability be $\eta=0.1,0.5$, and 0.9 , corresponding to a smaller, moderate, and larger amount of zero/one values, respectively. For a single covariate $X_{1}$ that follows the standard Gaussian distribution $N(0,1)$, we let the regression coefficients in the Bernoulli component be $\boldsymbol{\beta}=$ $\left(\beta_{0}, \beta_{1}\right)^{\prime}=(1,-1)^{\prime}$, and let the regression coefficients in the beta component be $\gamma=\left(\gamma_{0}, \gamma_{1}\right)^{\prime}=$ $(1,-1)^{\prime}$. The choice that $\beta_{1}$ and $\gamma_{1}$ have the same sign and magnitude allows comparison of the estimation accuracy of $\boldsymbol{\beta}$ and $\boldsymbol{\gamma}$ for different values of $\eta$. Let the precision parameter be $\phi=4$. We also let $\sigma^{2}=0.8$ and $\rho=0.6$, corresponding to a relatively strong spatial dependence. Next, data were generated under the spatial generalized Tobit model (22), where the cutoff point is set to $\alpha=3$, and the remaining simulation setup is the same.

Table 1 reports the properties of the parameter estimates for the spatial beta-Bernoulli mixture model by the mean estimates, relative biases, nominal standard deviations, mean standard errors, and mean squared errors, for different sample sizes $N$ and mixture probabilities $\eta$. For a given mixture probability $\eta$, as the sample size $N$ increases, the parameter estimates $\hat{\boldsymbol{\theta}}$ become more accurate, and the standard error estimates are closer to the nominal standard deviations. This indicates that $\hat{\boldsymbol{\theta}}$ and $\operatorname{vâr}(\hat{\boldsymbol{\theta}})$ are consistent estimates of $\boldsymbol{\theta}$ and $\operatorname{var}(\hat{\boldsymbol{\theta}})$, respectively. For a given sample size $N$, as the mixture probability $\eta$ increases, the data contain more zero/one values. In this case, the nominal standard deviations and standard errors of $\hat{\boldsymbol{\beta}}$ in the Bernoulli component become smaller, reflecting an increasing amount of information at zero/one, whereas those of $\hat{\gamma}$ and $\hat{\phi}$ in the beta component become larger. Overall, the simulation results indicate that the composite likelihood approach is able to recover the regression coefficients for both the Bernoulli and beta component, as well as the precision parameter and the dependence parameters, even when the sample size is relatively moderate. Similarly, for the spatial generalized Tobit model, Table 2 provides the mean estimates, relative biases, nominal standard deviations, mean standard errors, 
and mean squared errors for $N=900$ and $N=1600$. As the sample size $N$ increases, the parameter estimates $\hat{\boldsymbol{\theta}}$ become more accurate, and the standard errors are closer to the nominal standard deviations, which indicates the consistency of $\hat{\boldsymbol{\theta}}$ and $\operatorname{vâr}(\hat{\boldsymbol{\theta}})$ as before.

To compare the spatial beta-Bernoulli mixture model developed in Section 2 and the spatial generalized Tobit model in Section 4, we revisited the simulated data above. In particular, we considered the data generated from a spatial beta-Bernoulli model with $N=1600, \eta=0.1,0.5$, or 0.9 , and the remaining parameters $\left(\beta_{0}, \beta_{1}, \gamma_{0}, \gamma_{1}, \phi, \sigma^{2}, \rho\right)^{\prime}=(1,-1,1,-1,4,0.8,0.6)^{\prime}$. Then we fitted these data by a misspecified spatial generalized Tobit model with $\left(\alpha, \beta_{0}, \beta_{1}, \gamma_{0}, \gamma_{1}, \phi, \sigma^{2}, \rho\right)^{\prime}$ and a misspecified spatial Tobit model with parameters $\left(\alpha, \beta_{0}, \beta_{1}, \sigma^{2}, \rho\right)^{\prime}$. The parameter estimation results under these three models are given in Table 3 . The magnitudes of the correlation parameter estimate, $\hat{\rho}$, in the two misspecified models are close to the true value in the spatial beta-Bernoulli mixture model, while the variance component estimates $\left(\hat{\sigma}^{2}\right)$ underestimate $\sigma^{2}$. The parameter estimates under $\eta=0.9$ generally have less bias for all three models. The CLIC values obtained from fitting the correct spatial beta-Bernoulli model are smaller than the two misspecified models in all 100 simulations, while the discrepancy tends to be smaller for the spatial generalized Tobit model, indicating superiority over the existing spatial Tobit model.

For further comparison with the spatial generalized Tobit model and spatial Tobit model, we took one data set simulated from the spatial beta-Bernoulli mixture model, each with $N=1600$ and $\eta=0.1,0.5$ or 0.9 . Figure 2 gives the density plot of the actual simulated data from the spatial beta-Bernoulli mixture model, one density plot each for a data set simulated from the fitted correct model, the misspecified spatial generalized Tobit model, and the misspecified spatial Tobit model, for $\eta=0.1$ (upper left), $\eta=0.5$ (upper right), and $\eta=0.9$ (lower left). The density of the actual data has two peaks at both ends and the middle part is relatively flat. When $\eta$ increases from 0.1 to 0.5 and then to 0.9 , the density curves from the three fitted models become closer. This is expected, because in the extreme case of $\eta=1$, all three models are reduced to a spatial probit model for a binary response variable. On the other hand, when $\eta$ is relatively small, the spatial Tobit model does not adequately capture the density of data in the range between 0 and 1 . This is also expected, 
because the threshold mechanism in a spatial Tobit model will only transform the upper tail of the scaled Gaussian latent variable to one and the lower tail to zero, while the unimodal shape remains in the middle range between 0 and 1 . In contrast, the density curves from the fitted spatial betaBernoulli mixture model and the fitted spatial generalized Tobit model are much closer to the true curve. This example illustrates the greater flexibility of the new models proposed here that capture a wider variety of shapes of the data distributions.

We also considered the data simulated from the spatial generalized Tobit model and fitted them by the spatial beta-Bernoulli mixture model and spatial Tobit model as two misspecified models. For sample size $N=1600$, we set the true parameters to be $\left(\alpha, \beta_{0}, \beta_{1}, \gamma_{0}, \gamma_{1}, \phi, \sigma^{2}, \rho\right)^{\prime}=$ $(3,1,-1,1,-1,4,0.8,0.6)^{\prime}$. The parameter estimation results under these three models are given in Table 4 . The proportion of zero/one values in the simulated data is about $32 \%$, which is reflected in $\hat{\eta}=0.316$ in the spatial beta-Bernoulli mixture model. Although the interpretation of the regression parameters $\hat{\beta}_{1}$ and $\hat{\gamma}_{1}$ differs from the mixture model, the signs are the same as those of the true parameters. As before, the variance component estimate $\hat{\sigma}^{2}$ underestimates $\sigma^{2}$ and the intercept estimate $\hat{\beta}_{0}$ is quite different from the true parameter. Since the spatial Tobit model is only a special case of the spatial generalized Tobit model, the estimates have relatively larger biases compared to those under the correct model. The CLIC values obtained from the correct spatial generalized Tobit model are smaller than the two misspecified models in all 100 simulations.

To further compare with the spatial beta-Bernoulli mixture model and the spatial Tobit model, we took one simulated data set from the spatial generalized Tobit model. The lower right figure in Figure 2 gives the density curve of the actual simulated data from the spatial generalized Tobit model, one density plot each for a simulated dataset from the fitted correct model and the fitted misspecified models. It shows that the fitted correct model is closest to the density curve of the actual data. The fitted spatial beta-Bernoulli mixture model has a density curve that is much closer to the truth than the spatial Tobit model, again demonstrating the flexibility of the models developed here.

Finally, we considered two covariates $X_{1}$ and $X_{2}$ from the standard Gaussian distribution. For 
both the spatial beta-Bernoulli mixture and the spatial generalized Tobit models, we set the percentage of zero/one values close to the land cover data example. In the spatial beta-Bernoulli mixture model, the vector of regression coefficients for the beta component is set as $\boldsymbol{\beta}=\left(\beta_{0}, \beta_{1}, \beta_{2}\right)^{\prime}=$ $(2,0,1)^{\prime}$, and the vector of regression coefficients for the Bernoulli component is $\gamma=\left(\gamma_{0}, \gamma_{1}, \gamma_{2}\right)^{\prime}=$ $(1,-0.5,0)^{\prime}$. Under this setup, only $X_{2}$ affects the beta component, and only $X_{1}$ affects the Bernoulli component, representing two distinct different processes in the two mixture components. We set $\eta=0.1$ so that the percentage of zero/one values is exactly $10 \%$. The precision parameter is $\phi=1$, and the dependence parameters are $\left(\sigma^{2}, \rho\right)^{\prime}=(0.8,0.6)^{\prime}$. In the spatial generalized Tobit model, the parameters are set as $\alpha=4, \boldsymbol{\beta}=\left(\beta_{0}, \beta_{1}, \beta_{2}\right)^{\prime}=(1.5,-0.5,0)^{\prime}$, $\gamma=\left(\gamma_{0}, \gamma_{1}, \gamma_{2}\right)^{\prime}=(0.5,0,0)^{\prime}, \phi=1$, and $\left(\sigma^{2}, \rho\right)^{\prime}=(0.8,0.6)^{\prime}$. Under these parameter choices, the percentage of zero/one values is around 10\%, and the inverse beta CDF transformation is $\operatorname{Beta}(1 /(1+\exp (-0.5)), 1)$ across all sampling sites. Similar to the case of a single covariate, the results in Tables 1 and 2 show that both the parameter estimates and the variance estimates are consistent. When the data have a relatively low (or high) proportions of zero/one values, the

standard errors of $\hat{\boldsymbol{\beta}}$ in the Bernoulli component (or $\hat{\gamma}$ in the beta component) can be elevated. In contrast, for the spatial generalized Tobit model, the standard deviations and standard errors of $\hat{\boldsymbol{\beta}}$ and $\hat{\gamma}$ are comparable, although the standard errors of $\hat{\gamma}$ may also be large when the amount of proportions on $(0,1)$ low.

\section{Data Example}

\subsection{Study Background}

In the motivating data example, the spatial unit of analysis is a quarter section (or, 1/36th of a township, 160 acres $\approx 65$ ha). There are a total of $N=1429$ quarter sections in the study area in northern Wisconsin, USA (Figure 11). Forest cover types were derived from the Wisconsin Land Economic Inventory, a land survey that represents land covers in 1930. Here we consider an early successional forest cover, known as the aspen-paper birch (APB) (Fu et al., 2013; Feng et al. 2014). The response variable is the proportion of APB in a quarter section, varying from 0 
(no APB) to 1 (all APB). The covariates are about land-ownership characteristics derived from historical plat maps in 1915, as land ownership offers a spatially specific, quantifiable way to assess the influence of anthropogenic and historical factors on landscape structure. In this study, we consider the binary variable reservation that indicates whether the quarter section is on an Indian reservation; the number of parcels in a given quarter section which is a measure of parcel density; the proportion of the average parcel size as a measure of parcel size within a quarter section; the proportion of the largest parcel which reports the largest parcel polygon as a quarter section proportion; the average size of all parcels as a measure of all parcels that intersect with a quarter section; and total ownership area which shows the total property area throughout the project study area of the dominant owner of the quarter section. We took a natural logarithm transformation of total ownership area since the values of total area are skewed to the right. All the covariates except the binary variable reservation were centered and scaled to have sample mean 0 and sample variance 1.

\subsection{Model Fitting and Selection}

To assess the influence of land-ownership characteristics in 1915 to APB in 1930, we applied the spatial beta-Bernoulli mixture model and the spatial generalized Tobit model to analyze the data. We also fitted the spatial Tobit model for comparison. The weight radius is set to be $r=5$ (Feng et al., 2014). Maximum likelihood estimation assuming independence provided the initial values for composite likelihood estimation. Table 5 presents the parameter estimates and their standard errors, CLIC values, and computational time under each model. By a backward elimination, the final spatial generalized Tobit model has slightly lower CLIC $(-0.688)$ than the final mixture model $(-0.676)$. The common covariates retained in the two final models are reservation and average size of all parcels (Figure 1). After we excluded the insignificant covariate number of parcels and refitted the spatial generalized Tobit model, the CLIC is -0.681 , which is comparable to the mixture model.

For the spatial beta-Bernoulli mixture model, the mixture probability is estimated to be $\hat{\eta}=$ 0.12 , which agrees well with the observed $12 \%$ of quarter sections with no or all APB. For the 
regression coefficients in the Bernoulli component, the estimates are not significant except $\hat{\beta}_{1}$ for the binary variable reservation. This may be because we only have a relatively small amount of zero/one values, and the standard errors can be inflated as shown in the simulation results Table 1 . For the regression coefficients in the beta component, there is an additional significant covariate average size of all parcels $\left(\hat{\gamma}_{5}\right)$ besides reservation $\left(\hat{\gamma}_{1}\right)$. The first-order correlation $\sigma^{2} \rho$ is estimated to be $0.904 \times 0.502=0.454$. The results provide evidence that after accounting for the other covariates, the quarter sections on the reservation have larger proportions, including $100 \%$, of APB than off the reservation, supporting previous studies (Steen-Adams et al., 2007, 2011). In addition, the off-reservation quarter sections with higher average parcel size tend to have larger proportions of APB.

For the spatial generalized Tobit model, both reservation $\left(\hat{\beta}_{1}\right)$ and average size of all parcels $\left(\hat{\beta}_{5}\right)$ in the Gaussian latent variable model are significant. The results are in agreement with those from the spatial beta-Bernoulli mixture model. However, the interpretation is in terms of the latent variables instead of the original response variables. As for the inverse beta CDF transformation, the estimates are not significant except for the intercept $\hat{\gamma}_{0}$. That is, the inverse beta CDF transformation of the spatial latent variable does not depend on covariates. The first-order correlation $\sigma^{2} \rho$ is $0.855 \times 0.641=0.548$, which is higher than that of the spatial beta-Bernoulli model. Compared with the two proposed models, the spatial Tobit model has an additional significant covariate total

ownership area $\left(\hat{\beta}_{6}\right)$, although the small standard error estimate may be misleading as indicated in the simulation study. The first-order correlation is about 0.533 , which is close to the spatial generalized Tobit model, as demonstrated in the simulation example.

\subsection{Prediction and Model Comparison}

We now compare the models by prediction. First, we split the data into a training set (70\%) and a test set (30\%). Let $Y_{0}$ denote the response at say $\boldsymbol{s}_{0}$ in the test set. We predicted $Y_{0}$ by a conditional expectation

$$
E\left(Y_{0} \mid \boldsymbol{Y}_{T}\right)=P\left(Y_{0}=1 \mid \boldsymbol{Y}_{T}\right)+\int_{0}^{1} y_{0} \partial P\left(Y_{0} \leq y_{0} \mid \boldsymbol{Y}_{T}\right) / \partial y_{0} d y_{0}
$$


where $\boldsymbol{Y}_{T}$ denotes the vector of the training data. However, the computation of (25) is not straightforward. Let $N_{R}$ denote the number of proportional responses within $R=10$ units and $\boldsymbol{Y}_{N_{R}}$ denote the corresponding training data. The conditional expectation (25) was approximated by replacing $P\left(Y_{0}=1 \mid \boldsymbol{Y}_{T}\right)$ with $P\left(Y_{0}=1 \mid \boldsymbol{Y}_{N_{R}}\right)=\eta \int_{-\boldsymbol{x}_{0}^{\prime} \boldsymbol{\beta}}^{+\infty} \phi_{N_{R}+1}\left(y_{0}^{*}-\boldsymbol{x}_{0}^{\prime} \boldsymbol{\beta}, \boldsymbol{t}_{N_{R}} ; \boldsymbol{\Sigma}_{N_{R}+1}\right) d y_{0}^{*} / \phi_{N_{R}}\left(\boldsymbol{t}_{N_{R}} ; \boldsymbol{\Sigma}_{N_{R}}\right)$ and replacing $\partial P\left(Y_{0} \leq y_{0} \mid \boldsymbol{Y}_{T}\right) / \partial y_{0}$ with

$$
\begin{aligned}
\frac{\partial}{\partial y_{0}} P\left(Y_{0} \leq y_{0} \mid \boldsymbol{Y}_{N_{R}}\right)= & (1-\eta) \phi_{N_{R}+1}\left(\Phi_{1}^{-1}\left(F_{\text {Beta }}\left(y_{0} ; \boldsymbol{\gamma}, \phi\right)\right), \boldsymbol{t}_{N_{R}} ; \boldsymbol{\Sigma}_{N_{R}+1}\right) F_{\text {Beta }}\left(y_{0} ; \boldsymbol{\gamma}, \phi\right) \\
& \times\left\{\phi_{N_{R}}\left(\boldsymbol{t}_{N_{R}} ; \boldsymbol{\Sigma}_{N_{R}}\right) \phi_{1}\left(\Phi_{1}^{-1}\left(F_{\text {Beta }}\left(y_{0} ; \boldsymbol{\gamma}, \phi\right)\right)\right)\right\}^{-1}
\end{aligned}
$$

where $\boldsymbol{t}_{N_{R}}=\left(\Phi_{1}^{-1}\left(F_{\text {Beta }}\left(Y_{1} ; \boldsymbol{\gamma}, \phi\right)\right), \ldots, \Phi_{1}^{-1}\left(F_{\text {Beta }}\left(Y_{N_{R}} ; \boldsymbol{\gamma}, \phi\right)\right)\right)^{\prime}, \boldsymbol{\Sigma}_{N_{R}}$ is an $N_{R} \times N_{R}$ the covariance matrix of $\boldsymbol{t}_{N_{R}}$, and $\boldsymbol{\Sigma}_{N_{R}+1}$ is an $\left(N_{R}+1\right) \times\left(N_{R}+1\right)$ covariance matrix of $\left(y_{0}^{*}, \boldsymbol{t}_{N_{R}}^{\prime}\right)^{\prime}$. Further $\phi_{N_{R}}$ and $\phi_{N_{R}+1}$ denote the $N_{R}$-variate and $N_{R}+1$-variate Gaussian density functions.

Similarly, the conditional expectation under the spatial generalized Tobit model was approximated by

$$
E\left(Y_{0} \mid \boldsymbol{Y}_{N_{R}}\right)=P\left(Y_{0}=1 \mid \boldsymbol{Y}_{N_{R}}\right)+\int_{0}^{1} y_{0} \partial P\left(Y_{0} \leq y_{0} \mid \boldsymbol{Y}_{N_{R}}\right) / \partial y_{0} d y_{0}
$$

where $P\left(Y_{0}=1 \mid \boldsymbol{Y}_{N_{R}}\right)=\int_{\alpha-\boldsymbol{x}_{0}^{\prime} \boldsymbol{\beta}}^{+\infty} \phi_{N_{R}+1}\left(y_{0}^{*}-\boldsymbol{x}_{0}^{\prime} \boldsymbol{\beta}, \boldsymbol{t}_{N_{R}} ; \boldsymbol{\Sigma}_{N_{R}+1}\right) d y_{0}^{*} / \phi_{N_{R}}\left(\boldsymbol{t}_{N_{R}} ; \boldsymbol{\Sigma}_{N_{R}}\right), \boldsymbol{t}_{N_{R}}=$ $\left(\alpha F_{\text {Beta }}\left(Y_{1} ; \boldsymbol{\gamma}, \phi\right), \ldots, \alpha F_{\text {Beta }}\left(Y_{N_{R}} ; \boldsymbol{\gamma}, \phi\right)\right)^{\prime}-\boldsymbol{X}_{N_{R}} \boldsymbol{\beta}, \boldsymbol{X}_{N_{R}}$ is the design matrix corresponding to $\boldsymbol{Y}_{N_{R}}$, and $\frac{\partial}{\partial y_{0}} P\left(Y_{0} \leq y_{0} \mid \boldsymbol{Y}_{N_{R}}\right)=\alpha f_{\text {Beta }}\left(y_{0} ; \boldsymbol{\gamma}, \phi\right) \phi_{N_{R}+1}\left(\alpha F_{\text {Beta }}\left(y_{0} ; \boldsymbol{\gamma}, \phi\right)-\boldsymbol{x}_{0}^{\prime} \boldsymbol{\beta}, \boldsymbol{t}_{N_{R}} ; \boldsymbol{\Sigma}_{N_{R}+1}\right) / \phi_{N_{R}}\left(\boldsymbol{t}_{N_{R}} ; \boldsymbol{\Sigma}_{N_{R}}\right)$.

The conditional expectation under the spatial Tobit model was approximated by

$$
E\left(Y_{0} \mid \boldsymbol{Y}_{N_{R}}\right)=P\left(Y_{0}=1 \mid \boldsymbol{Y}_{N_{R}}\right)+\int_{0}^{1} y_{0} \partial P\left(Y_{0} \leq y_{0} \mid \boldsymbol{Y}_{N_{R}}\right) / \partial y_{0} d y_{0}
$$


where

$$
\begin{aligned}
& P\left(Y_{0}=1 \mid \boldsymbol{Y}_{N_{R}}\right)=\alpha^{N_{R}} \int_{\alpha}^{+\infty} f\left(y_{0}^{*} \mid \alpha \boldsymbol{Y}_{N_{R}}\right) d y_{0}^{*} \\
&=\alpha^{N_{R}} \int_{\alpha}^{+\infty} f\left(y_{0}^{*}, \alpha \boldsymbol{Y}_{N_{R}}\right) d y_{0}^{*} / \phi_{N_{R}}\left(\boldsymbol{t}_{N_{R}} ; \boldsymbol{\Sigma}_{N_{R}}\right) \\
&=\alpha^{N_{R}} \int_{\alpha-\boldsymbol{x}_{0}^{\prime} \boldsymbol{\beta}}^{+\infty} \phi_{N_{R}+1}\left(y_{0}^{*}-\boldsymbol{x}_{0}^{\prime} \boldsymbol{\beta}, \boldsymbol{t}_{N_{R}} ; \boldsymbol{\Sigma}_{N_{R}+1}\right) d y_{0}^{*} / \phi_{N_{R}}\left(\boldsymbol{t}_{N_{R}} ; \boldsymbol{\Sigma}_{N_{R}}\right), \\
& \frac{\partial}{\partial y_{0}} P\left(Y_{0} \leq y_{0} \mid \boldsymbol{Y}_{N_{R}}\right)=\alpha^{N_{R}+1} \phi_{N_{R}+1}\left(y_{0}-\boldsymbol{x}_{0}^{\prime} \boldsymbol{\beta}, \boldsymbol{t}_{N_{R}} ; \boldsymbol{\Sigma}_{N_{R}+1}\right) / \phi_{N_{R}}\left(\boldsymbol{t}_{N_{R}} ; \boldsymbol{\Sigma}_{N_{R}}\right) \text { for } y_{0} \in(0,1), \\
& \text { and } \boldsymbol{t}_{N_{R}}=\alpha \boldsymbol{Y}_{N_{R}}-\boldsymbol{X}_{N_{R}} \boldsymbol{\beta} .
\end{aligned}
$$

The mean absolute error (MAE) and the root mean squared error (RMSE) were computed to evaluate the prediction performance. The MAE is defined as $n^{-1} \sum_{i=1}^{n}\left|y_{i}-\hat{y}_{i}\right|$, where $n$ is the sample size of the training or test data, $y_{i}$ is the observation, and $\hat{y}_{i}$ is the prediction based on conditional expectation. The MAE is an average of absolute differences and is not sensitive to unusually large errors. In contrast, RMSE, defined as $\left\{n^{-1} \sum_{i=1}^{n}\left(y_{i}-\hat{y}_{i}\right)^{2}\right\}^{1 / 2}$, is based on an average of squared differences and is more sensitive to large errors.

Figures 3 and 4 present the prediction results for the spatial beta-Bernoulli mixture model, spatial generalized Tobit model, and the spatial Tobit model. The scatter plots in Figure 3 show that the two new models slightly overfit zero/one values, while the spatial Tobit model underfit them. Our additional simulation results indicate that the overfitting in the data example was more due to random chances (results not shown). In Figure 4 , the three models have similar patterns of predictions across sampling sites. Compared with the two new models, the spatial Tobit model tends to have more smoothed predictions, especially at sites with zero/one values, as observed in Figure 3 . For the training data, the smallest RMSE and MAE are obtained for the spatial generalized Tobit model, with 0.107 and 0.071 , respectively. In addition, the RMSE of the spatial beta-Bernoulli mixture model is about $12 \%$ higher than the spatial Tobit model, while the MAEs are about the same. For the test data, the spatial Tobit model has the smallest RMSE and the spatial generalized Tobit model has the smallest MAE (Table 6). 
Figure 5 presents the density plot of the observed APB, as well as the density curves based on 100 simulated datasets from each of the fitted spatial beta-Bernoulli mixture model, fitted spatial generalized Tobit model, fitted spatial Tobit model, and the final fitted spatial generalized Tobit model. For the spatial Tobit model, the unimodal shape in the open interval $(0,1)$ is restrictive, while for all the other models, the density curves from the simulated datasets are quite close to the that from the observed data.

In terms of the computational time, the spatial Tobit model took about 1 minute on a 64 bit Linux operating system with 48-64 GB of RAM, and the spatial beta-Bernoulli mixture and generalized spatial Tobit model took about 30 minutes, which is a moderately high price paid for the model flexibility. The additional computational cost in the latter two models can be attributed largely to the numerical score functions in the optimization procedure instead of the closed-form score function as in the spatial Tobit model.

\section{Conclusions and Discussion}

In this paper, we have developed a beta-Bernoulli mixture model and a spatial generalized Tobit model for spatial proportional data with zero/one values. With a composite likelihood approach, numerical examples have shown that both the regression coefficients and the dependence parameters can be estimated consistently. Moreover, the composite likelihood estimation developed here is relatively efficient to compute and thus, is applicable for spatial data with a relatively large sample size. Both the simulation study and the data example have shown that the proposed spatial beta-Bernoulli mixture model and spatial generalized Tobit model are more flexible than the existing spatial Tobit model, while the cost for this added flexibility is primarily computational.

In both new models, the Gaussian latent variables provide a way to account for the spatial dependence among observations. The spatial beta-Bernoulli mixture model assumes that the data are generated from two distributions, one for the zero/one values with mean $\nu_{i}$ in relation to covariates via $\boldsymbol{\beta}$ and the other one for the proportions with mean $\mu_{i}$ in relation to covariates via $\gamma$. The expectation of the response variable is given by $\mathrm{E}\left(Y_{i}\right)=\eta \nu_{i}+(1-\eta) \mu_{i}$ and the interpretation 
is straightforward. With the spatial generalized Tobit model, the data are governed by the spatial Gaussian latent variables with model flexibility enhanced by an inverse beta CDF transformation. The response variable does not follow a known distribution and the expectation of the response variable $\mathrm{E}\left(Y_{i}\right)$ is not straightforward to derive. However, the expectation of the response variable increases as the mean of the spatial latent variable $\boldsymbol{x}_{i}^{\prime} \boldsymbol{\beta}$ and the mean of beta random variable $\mu_{i}$ increase and thus, the interpretation is also intuitive.

The numerical examples show that the spatial generalized Tobit model appears to be as flexible as the spatial beta-Bernoulli mixture model, and the computational cost for the two proposed models is comparable. In practice, if the zero/one values are due to thresholding such as a detection limit, a spatial Tobit model can be applied first. When there remains a large discrepancy between the fitted and the actual observations, a spatial generalized Tobit model can be considered. If the zero/one values and proportions are associated with different factors, one could consider a twostage approach where the first stage determines whether the response is zero/one or a proportion, and the second stage models the proportional data on $(0,1)$ with a beta distribution excluding the zero/one values. The mixture model has the advantage of accounting for the two processes in a single model and stage.

Common to both the mixture and Tobit type of models is the specification of a spatial latent variable. A practical concern is whether the parameter estimates change significantly if the latent distribution is not Gaussian. The robustness of latent variable models has been discussed for correlated binary data by Tan et al. (1999). Liu (2004) introduced the "robit regression" using a latent T-distribution as a more robust alternative to the traditional logit and probit models. It would be interesting to evaluate how robust the parameter estimates and predictions are in our models. In addition, for spatial compositional data with zero values, Leininger et al. (2013) proposed a Bayesian hierarchical model by a power scaling transformation to model the presence of zeros in compositional data, under the assumption that there exists at least one nonzero component for all observations. The composite likelihood approach may also be extended to models for spatial compositional data. We leave these for future research. 


\section{Acknowledgments}

Funding has been provided for this research from a USDA Cooperative State Research, Education and Extension Service (CSREES) McIntire-Stennis project and the National Science Foundation PalEON MacroSystems Biology under grant no. DEB1241868. We also thank the editor, an associate editor, and two referees for constructive comments that improved the paper.

\section{References}

Akaike, H. (1973). Information theory and an extension of the maximum likelihood principle. Second International Symposium on Information Theory, B. N. Petrov and F. Csaki (Eds.), 267281. Akademia Kiado, Budapest.

Apanasovich, T. V., Ruppert, D., Lupton, J. R., Popovic, N., Turner, N. D., Chapkin, R. S., and Carroll, R. J. (2008). Aberrant crypt foci and semiparametric modeling of correlated binary data. Biometrics, 64, 490-500.

Byrd, R. H., Lu, P., Nocedal, J., and Zhu, C. (1995). A limited memory algorithm for bound constrained optimization. SIAM Journal on Scientific Computing, 16, 1190-1208.

De Oliveira, V. (2000). Bayesian prediction of clipped Gaussian random fields. Computational Statistics and Data Analysis, 34, 299-314.

Eskelson, B. N., Madsen, L., Hagar, J. C., and Temesgen, H. (2011). Estimating riparian understory vegetation cover with beta regression and copula models. Forest Science, 57, 212-221.

Ferrari, S.L. and Cribari-Neto, F. (2004). Beta regression for modelling rates and proportions. Journal of Applied Statistics, 31, 799-815.

Feng, X., Zhu, J., Steen-Adams, M. M., and Lin, P. S. (2014). Composite likelihood estimation for models of spatial ordinal data and spatial proportional data with zero/one values. Environmetrics, 25, 571-583. 
Fu, R., Thurman, A. L., Chu, T., Steen-Adams, M. M., and Zhu, J. (2013). On estimation and selection of autologistic regression models via penalized pseudolikelihood. Journal of Agricultural, Biological, and Environmental Statistics, 18, 429-449.

Godambe, V. P. (1960). An optimum property of regular maximum likelihood estimation. Annals of Mathematical Statistics, 31, 1208-1211.

Guolo, A. and Varin, C. (2014). Beta regression for time series analysis of bounded data, with application to Canada Google ${ }^{\circledR}$ Flu Trends. The Annals of Applied Statistics, 8, 74-88.

Guyon, X. (1995). Random Fields on a Network: Modeling, Statistics, and Applications. Springer, New York.

Heagerty, P. J. and Lele, S. R. (1998). A composite likelihood approach to binary spatial data. Journal of the American Statistical Association, 93, 1099-1111.

Kieschnick, R. and McCullough, B. D. (2003). Regression analysis of variates observed on (0, 1): percentages, proportions and fractions. Statistical Modelling, 3, 193-213.

Leininger, T. J., Gelfand, A. E., Allen, J. M., and Silander Jr., J. A. (2013). Spatial regression modeling for compositional data with many zeros. Journal of Agricultural, Biological, and Environmental Statistics, 18, 314-334.

Lindsay, B. (1988). Composite likelihood methods. Contemporary Mathematics, 80, 221-239.

Liu, C. (2004). Robit regression: a simple robust alternative to logistic and probit regression. Applied Bayesian Modeling and Causal Inference from Incomplete-data Perspectives, Gelman A, Meng XL (Eds.), 227-238. Wiley, New York.

Mielke Jr, P. W. (1975). Convenient beta distribution likelihood techniques for describing and comparing meteorological data. Journal of Applied Meteorology, 14, 985-990.

Ospina, R. and Ferrari, S. L. (2010). Inflated beta distributions. Statistical Papers, 51, 111-126. 
Ospina, R. and Ferrari, S. L. (2012). A general class of zero-or-one inflated beta regression models. Computational Statistics and Data Analysis, 56, 1609-1623.

Rao, T. S. (1970). The fitting of non-stationary time-series models with time-dependent parameters. Journal of the Royal Statistical Society, Series B, 32, 312-322.

Simas, A. B., Barreto-Souza, W., and Rocha, A. V. (2010). Improved estimators for a general class of beta regression models. Computational Statistics and Data Analysis, 54, 348-366.

Steen-Adams, M. M., Langston, N., and Mladenoff, D. J. (2007). White pine in the northern forests: an ecological and management history of white pine on the Bad River Reservation of Wisconsin. Environmental History, 12, 614-648.

Steen-Adams, M. M., Mladenoff, D. J., Langston, N. E., Liu, F., and Zhu, J. (2011). Influence of biophysical factors and differences in Ojibwe reservation versus Euro-American social histories on forest landscape change in northern Wisconsin, USA. Landscape Ecology, 26, 1165-1178.

Tan, M., Qu, Y., and Sunil Rao, J. (1999). Robustness of the latent variable model for correlated binary data. Biometrics, 55, 258-263.

Varin, C., Reid, N., and Firth, D. (2011). An overview of composite likelihood methods. Statistica Sinica, 21, 5-42.

Varin, C. and Vidoni, P. (2005). A note on composite likelihood inference and model selection. Biometrika, 92, 519-528. 


\section{Tables and Figures}
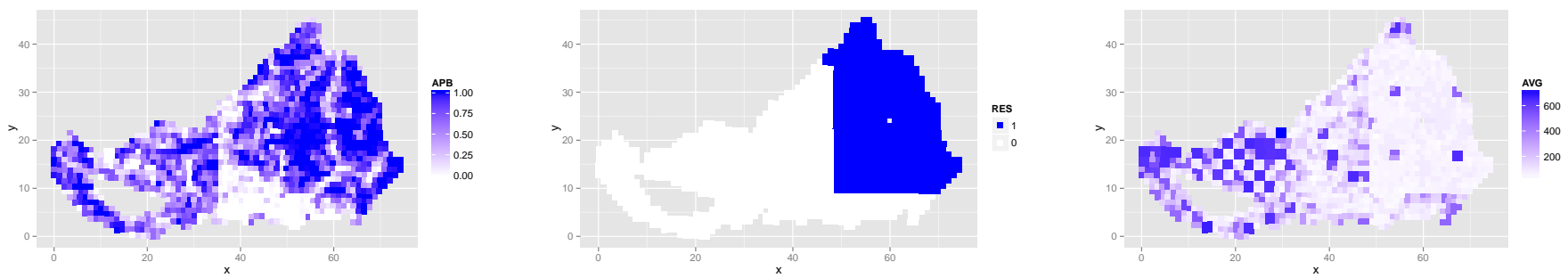

Figure 1: Data example: maps of the proportions of aspen-paper birch (APB) (left), reservation (middle), and average size of all parcels (right) in $N=1429$ quarter sections of a study area in northern Wisconsin.

$\eta=0.1$

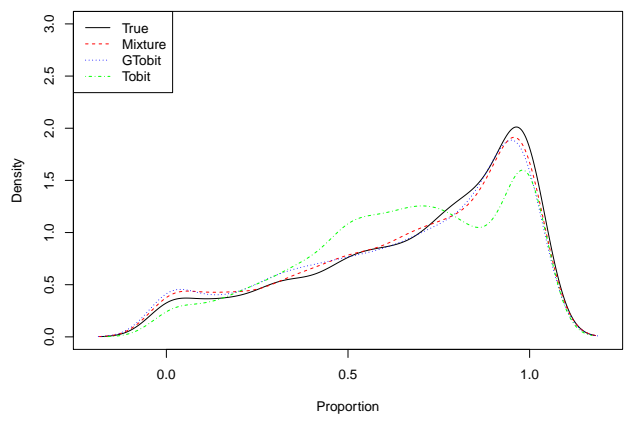

$\eta=0.9$

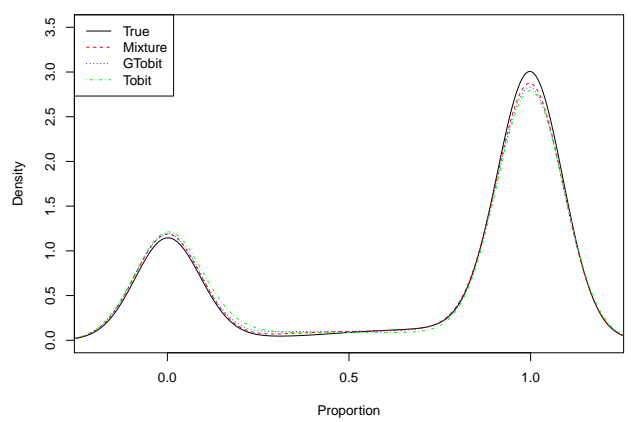

$\eta=0.5$

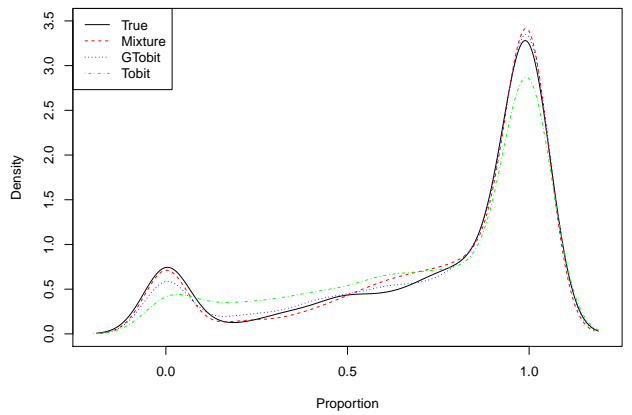

$\alpha=3$

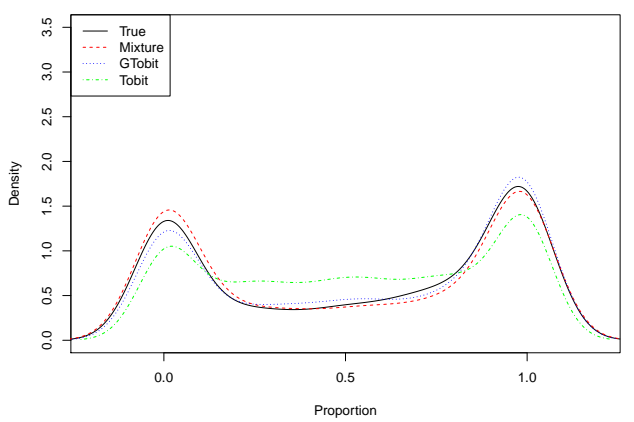

Figure 2: Simulation study: density plots of observed and fitted data from one simulation under the true spatial beta-Bernoulli mixture model with the mixture probability $\eta=0.1$ (upper left), 0.5 (upper right), 0.9 (lower left) and under the true spatial generalized Tobit model (lower right) with the cutoff point $\alpha=3$. The sample size is

$$
N=1600 \text {. }
$$



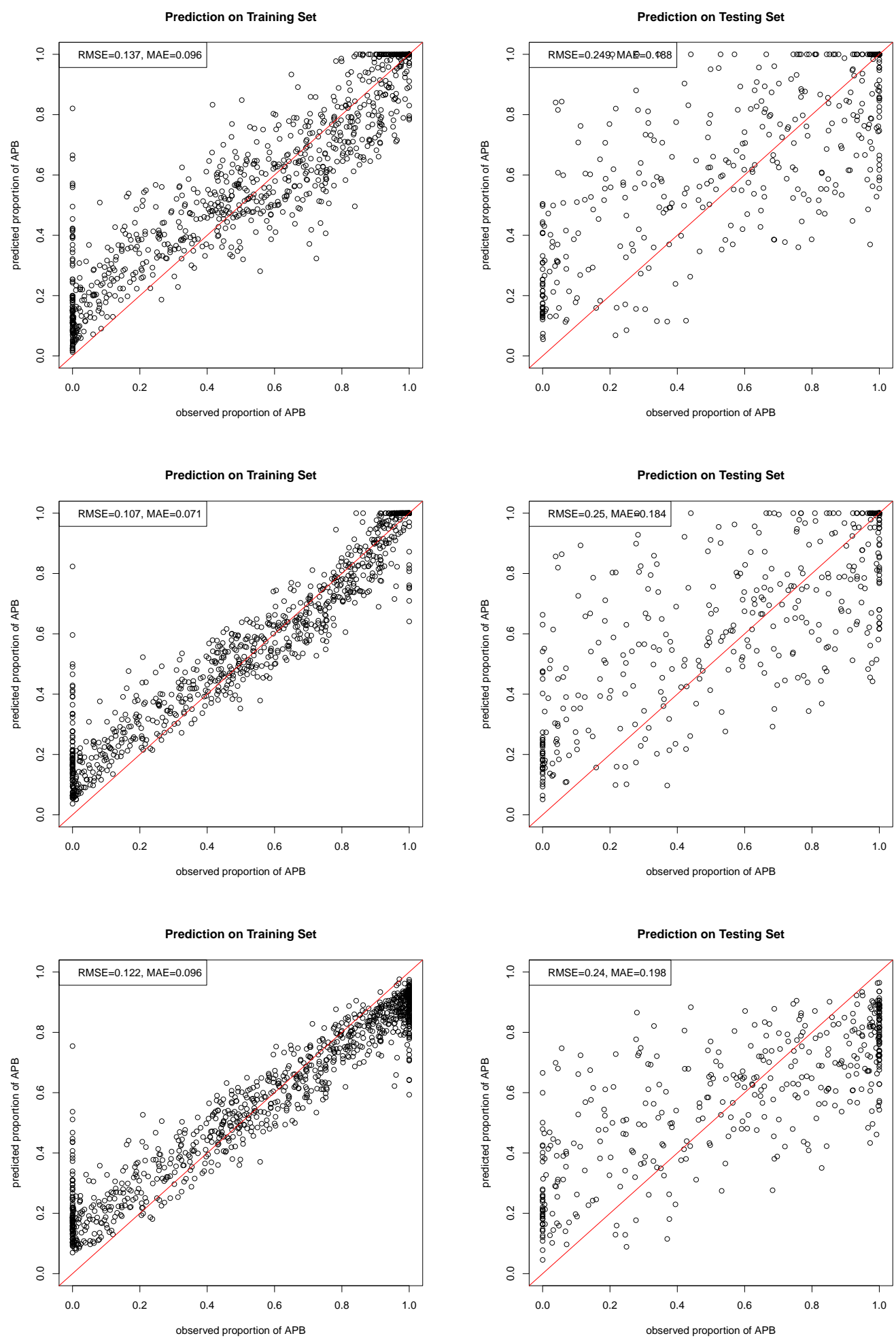

Figure 3: Data example: predictions versus observations under spatial beta-Bernoulli mixture model (row 1), spatial generalized Tobit model (row 2), and spatial Tobit model (row 3) for $70 \%$ training data (left column) and $30 \%$ testing data (right column). 

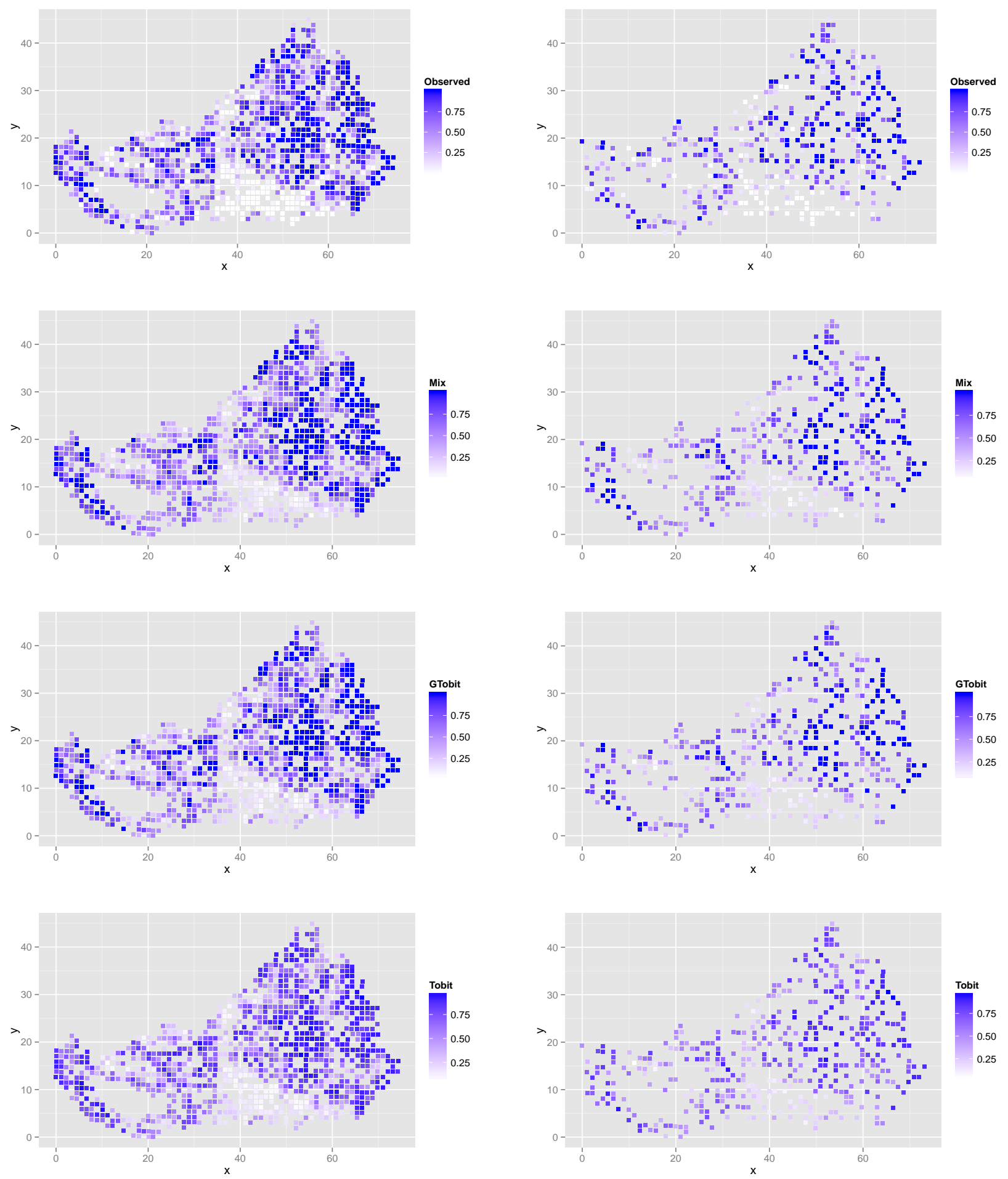

Figure 4: Data example: observations (row 1) and predictions from spatial beta-Bernoulli mixture model (row 2), spatial generalized Tobit model (row 3), and spatial Tobit model (row 4) for $70 \%$ training data (left column) and $30 \%$ testing data (right column). 

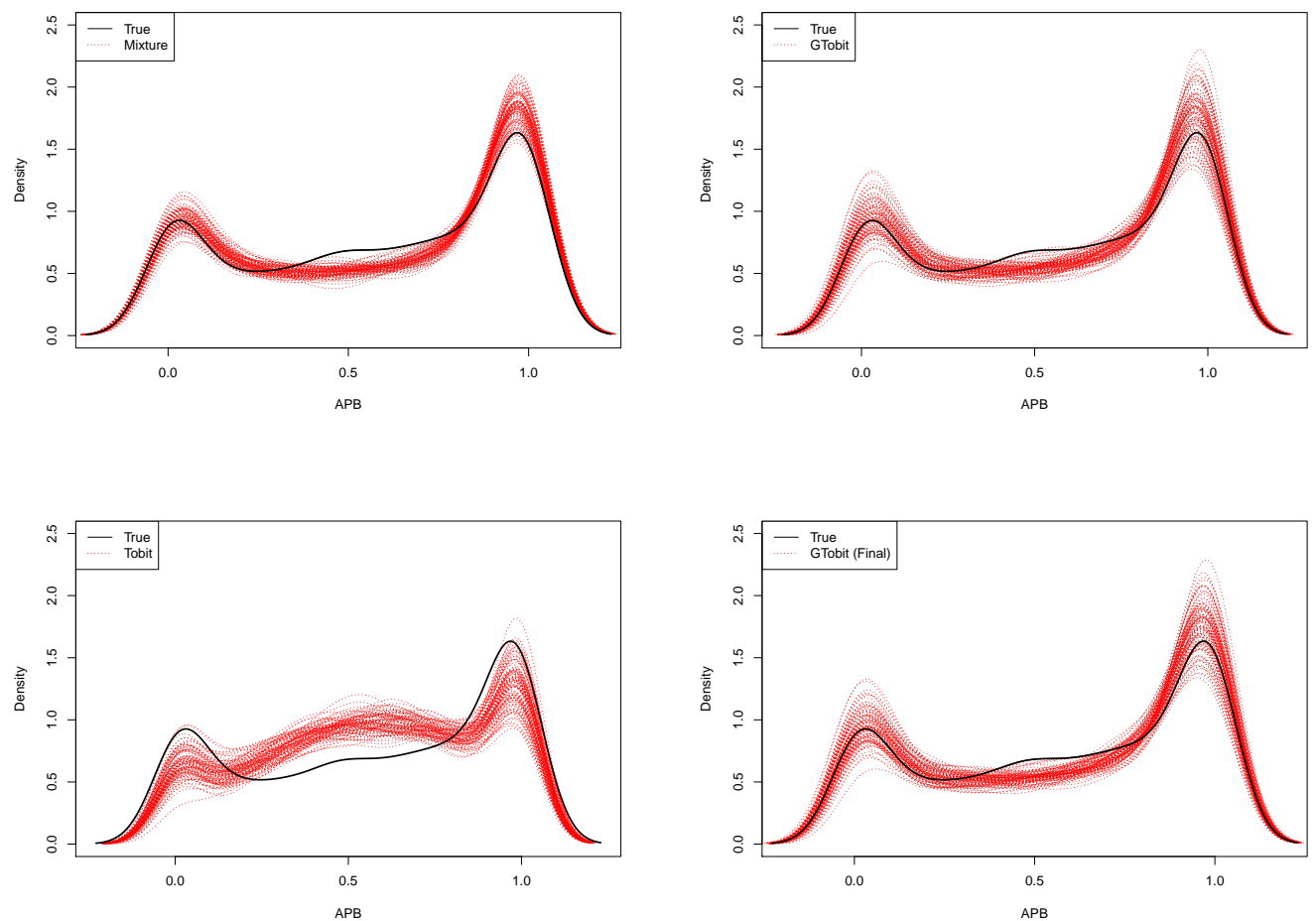

Figure 5: Data example: comparison between the density curves of the observed data and 100 simulated datasets from the fitted spatial beta-Bernoulli mixture model (upper left), fitted spatial generalized Tobit model (upper right), fitted spatial Tobit model (lower left), and final spatial generalized Tobit model (lower right). 
Table 1: Simulation study: composite likelihood estimates for the parameters of a spatial beta-Bernoulli mixture model: mean estimates (Estimate), relative biases (RB), nominal standard deviations (SD), mean standard errors (SE), and mean squared errors (MSE), with sample size $N=900$ or 1600 based on 100 simulations. $X_{1}, X_{2} \sim N(0,1)$.

\begin{tabular}{|c|c|c|c|c|c|c|c|c|c|c|}
\hline \multirow{2}{*}{ Parameter $N$} & \multicolumn{2}{|c|}{ Estimate } & \multicolumn{2}{|c|}{$\mathrm{RB}$} & \multicolumn{2}{|c|}{ SD } & \multicolumn{2}{|c|}{ SE } & \multicolumn{2}{|c|}{ MSE } \\
\hline & 900 & 1600 & 900 & 1600 & 900 & 1600 & 900 & 1600 & 900 & 1600 \\
\hline & \multicolumn{10}{|c|}{$\eta=0.1$, single covariate } \\
\hline$\eta=0.1$ & 0.100 & 0.100 & -0.003 & 0.002 & 0.010 & 0.007 & 0.010 & 0.008 & 0.000 & 0.000 \\
\hline$\beta_{0}=1$ & 1.059 & 1.039 & 0.059 & 0.039 & 0.294 & 0.212 & 0.261 & 0.190 & 0.090 & 0.046 \\
\hline$\beta_{1}=-1$ & -1.063 & -1.060 & 0.063 & 0.060 & 0.291 & 0.210 & 0.260 & 0.190 & 0.089 & 0.048 \\
\hline$\sigma^{2}=0.8$ & 0.864 & 0.826 & 0.080 & 0.033 & 0.100 & 0.094 & 0.131 & 0.094 & 0.014 & 0.009 \\
\hline$\rho=0.6$ & 0.557 & 0.573 & -0.072 & -0.045 & 0.083 & 0.063 & 0.088 & 0.066 & 0.009 & 0.005 \\
\hline$\gamma_{0}=1$ & 1.026 & 0.997 & 0.026 & -0.003 & 0.132 & 0.096 & 0.129 & 0.096 & 0.018 & 0.009 \\
\hline$\gamma_{1}=-1$ & -1.003 & -0.973 & 0.003 & -0.027 & 0.044 & 0.029 & 0.039 & 0.029 & 0.002 & 0.002 \\
\hline \multirow[t]{2}{*}{$\phi=4$} & 4.125 & 4.173 & 0.031 & 0.043 & 0.393 & 0.284 & 0.391 & 0.291 & 0.170 & 0.110 \\
\hline & \multicolumn{10}{|c|}{$\eta=0.5$, single covariate } \\
\hline$\eta=0.5$ & 0.501 & 0.502 & 0.002 & 0.004 & 0.015 & 0.012 & 0.017 & 0.013 & 0.000 & 0.000 \\
\hline$\beta_{0}=1$ & 1.028 & 1.024 & 0.028 & 0.024 & 0.161 & 0.133 & 0.165 & 0.122 & 0.027 & 0.018 \\
\hline$\beta_{1}=-1$ & -1.022 & -1.012 & 0.022 & 0.012 & 0.101 & 0.081 & 0.115 & 0.084 & 0.011 & 0.007 \\
\hline$\sigma^{2}=0.8$ & 0.860 & 0.826 & 0.075 & 0.032 & 0.113 & 0.111 & 0.151 & 0.107 & 0.016 & 0.013 \\
\hline$\rho=0.6$ & 0.558 & 0.573 & -0.069 & -0.044 & 0.086 & 0.069 & 0.094 & 0.070 & 0.009 & 0.006 \\
\hline$\gamma_{0}=1$ & 1.033 & 1.014 & 0.033 & 0.014 & 0.134 & 0.097 & 0.134 & 0.102 & 0.019 & 0.010 \\
\hline$\gamma_{1}=-1$ & -1.002 & -1.002 & 0.002 & 0.002 & 0.054 & 0.039 & 0.051 & 0.038 & 0.003 & 0.002 \\
\hline \multirow[t]{2}{*}{$\phi=4$} & 4.147 & 4.130 & 0.037 & 0.033 & 0.439 & 0.310 & 0.435 & 0.320 & 0.214 & 0.113 \\
\hline & \multicolumn{10}{|c|}{$\eta=0.9$, single covariate } \\
\hline$\eta=0.9$ & 0.900 & 0.901 & 0.000 & 0.001 & 0.011 & 0.008 & 0.010 & 0.008 & 0.000 & 0.000 \\
\hline$\beta_{0}=1$ & 1.031 & 1.026 & 0.031 & 0.026 & 0.157 & 0.114 & 0.154 & 0.115 & 0.026 & 0.014 \\
\hline$\beta_{1}=-1$ & -1.015 & -1.008 & 0.015 & 0.008 & 0.085 & 0.062 & 0.090 & 0.066 & 0.008 & 0.004 \\
\hline$\sigma^{2}=0.8$ & 0.851 & 0.817 & 0.064 & 0.022 & 0.135 & 0.133 & 0.190 & 0.137 & 0.021 & 0.018 \\
\hline$\rho=0.6$ & 0.559 & 0.573 & -0.068 & -0.046 & 0.095 & 0.078 & 0.106 & 0.080 & 0.011 & 0.007 \\
\hline$\gamma_{0}=1$ & 1.029 & 1.007 & 0.029 & 0.007 & 0.161 & 0.112 & 0.167 & 0.124 & 0.027 & 0.013 \\
\hline$\gamma_{1}=-1$ & -0.992 & -1.006 & -0.008 & 0.006 & 0.124 & 0.091 & 0.112 & 0.084 & 0.015 & 0.008 \\
\hline \multirow[t]{2}{*}{$\phi=4$} & 4.339 & 4.234 & 0.085 & 0.058 & 0.785 & 0.608 & 0.741 & 0.530 & 0.731 & 0.424 \\
\hline & \multicolumn{10}{|c|}{$\eta=0.1$, two covariates } \\
\hline$\eta=0.1$ & 0.100 & 0.101 & 0.004 & 0.009 & 0.010 & 0.007 & 0.010 & 0.008 & 0.000 & 0.000 \\
\hline$\beta_{0}=2$ & 2.411 & 2.230 & 0.206 & 0.115 & 1.015 & 0.413 & 0.776 & 0.398 & 1.199 & 0.224 \\
\hline$\beta_{1}=0$ & -0.057 & -0.030 & - & - & 0.435 & 0.194 & 0.387 & 0.216 & 0.193 & 0.038 \\
\hline$\beta_{2}=1$ & 1.163 & 1.135 & 0.163 & 0.135 & 0.594 & 0.307 & 0.566 & 0.313 & 0.380 & 0.112 \\
\hline$\sigma^{2}=0.8$ & 0.861 & 0.828 & 0.077 & 0.035 & 0.102 & 0.094 & 0.117 & 0.084 & 0.014 & 0.010 \\
\hline$\rho=0.6$ & 0.557 & 0.573 & -0.071 & -0.045 & 0.082 & 0.063 & 0.078 & 0.059 & 0.009 & 0.005 \\
\hline$\gamma_{0}=1$ & 1.035 & 1.018 & 0.035 & 0.018 & 0.180 & 0.133 & 0.161 & 0.123 & 0.034 & 0.018 \\
\hline$\gamma_{1}=-0.5$ & -0.493 & -0.493 & -0.014 & -0.015 & 0.048 & 0.033 & 0.040 & 0.030 & 0.002 & 0.001 \\
\hline$\gamma_{2}=0$ & -0.011 & 0.000 & - & - & 0.040 & 0.036 & 0.034 & 0.025 & 0.002 & 0.001 \\
\hline$\phi=1$ & 1.036 & 1.036 & 0.036 & 0.036 & 0.097 & 0.069 & 0.094 & 0.070 & 0.011 & 0.006 \\
\hline
\end{tabular}


Table 2: Simulation study: omposite likelihood estimates for spatial generalized Tobit model: mean estimates (Estimate), relative biases (RB), nominal standard deviations (SD), mean standard errors (SE), and mean squared errors (MSE) with sample size $N=900$ or 1600 based on 100 simulations. $X_{1}, X_{2} \sim N(0,1)$.

\begin{tabular}{|c|c|c|c|c|c|c|c|c|c|c|}
\hline \multirow{2}{*}{ Parameter $N$} & \multicolumn{2}{|c|}{ Estimate } & \multicolumn{2}{|c|}{$\mathrm{RB}$} & \multicolumn{2}{|c|}{ SD } & \multicolumn{2}{|c|}{ SE } & \multicolumn{2}{|c|}{ MSE } \\
\hline & 900 & 1600 & 900 & 1600 & 900 & 1600 & 900 & 1600 & 900 & 1600 \\
\hline & \multicolumn{10}{|c|}{ single covariate } \\
\hline$\alpha=3$ & 3.033 & 3.044 & 0.011 & 0.015 & 0.169 & 0.118 & 0.188 & 0.142 & 0.030 & 0.016 \\
\hline$\beta_{0}=1$ & 1.033 & 1.029 & 0.033 & 0.029 & 0.149 & 0.111 & 0.160 & 0.123 & 0.023 & 0.013 \\
\hline$\beta_{1}=-1$ & -1.018 & -1.019 & 0.018 & 0.019 & 0.067 & 0.048 & 0.075 & 0.056 & 0.005 & 0.003 \\
\hline$\sigma^{2}=0.8$ & 0.871 & 0.828 & 0.088 & 0.035 & 0.099 & 0.093 & 0.150 & 0.110 & 0.015 & 0.009 \\
\hline$\rho=0.6$ & 0.555 & 0.573 & -0.076 & -0.045 & 0.082 & 0.063 & 0.102 & 0.080 & 0.009 & 0.005 \\
\hline$\gamma_{0}=1$ & 1.003 & 0.994 & 0.003 & -0.006 & 0.040 & 0.030 & 0.040 & 0.029 & 0.002 & 0.001 \\
\hline$\gamma_{1}=-1$ & -0.999 & -0.995 & -0.001 & -0.005 & 0.059 & 0.043 & 0.060 & 0.044 & 0.004 & 0.002 \\
\hline \multirow[t]{2}{*}{$\phi=4$} & 4.042 & 4.018 & 0.011 & 0.005 & 0.307 & 0.210 & 0.307 & 0.223 & 0.096 & 0.045 \\
\hline & \multicolumn{10}{|c|}{ two covariates } \\
\hline$\alpha=4$ & 4.060 & 4.056 & 0.015 & 0.014 & 0.251 & 0.179 & 0.264 & 0.198 & 0.067 & 0.035 \\
\hline$\beta_{0}=1.5$ & 1.541 & 1.533 & 0.028 & 0.022 & 0.177 & 0.120 & 0.175 & 0.134 & 0.033 & 0.015 \\
\hline$\beta_{1}=-0.5$ & -0.515 & -0.508 & 0.029 & 0.015 & 0.074 & 0.050 & 0.069 & 0.051 & 0.006 & 0.003 \\
\hline$\beta_{2}=0$ & -0.004 & -0.002 & - & - & 0.062 & 0.048 & 0.056 & 0.041 & 0.004 & 0.002 \\
\hline$\sigma^{2}=0.8$ & 0.866 & 0.829 & 0.082 & 0.037 & 0.100 & 0.089 & 0.143 & 0.107 & 0.014 & 0.009 \\
\hline$\rho=0.6$ & 0.555 & 0.572 & -0.075 & -0.046 & 0.083 & 0.061 & 0.101 & 0.079 & 0.009 & 0.004 \\
\hline$\gamma_{0}=0.5$ & 0.506 & 0.497 & 0.013 & -0.007 & 0.074 & 0.050 & 0.073 & 0.055 & 0.006 & 0.002 \\
\hline$\gamma_{1}=0$ & 0.004 & -0.002 & - & - & 0.062 & 0.047 & 0.061 & 0.045 & 0.004 & 0.002 \\
\hline$\gamma_{2}=0$ & -0.008 & 0.001 & - & - & 0.049 & 0.040 & 0.052 & 0.039 & 0.002 & 0.002 \\
\hline$\phi=1$ & 1.008 & 1.007 & 0.008 & 0.007 & 0.071 & 0.050 & 0.067 & 0.049 & 0.005 & 0.003 \\
\hline
\end{tabular}


Table 3: Simulation study: composite likelihood estimates under the misspecified generalized spatial Tobit model (GTobit) and spatial Tobit model (Tobit). The true model is the spatial beta-Bernoulli mixture model (Mixture) with sample size $N=1600$ based on 100 simulations.

\begin{tabular}{|c|c|c|c|c|c|c|c|c|c|}
\hline \multirow{2}{*}{ Parameter } & \multicolumn{3}{|c|}{ Estimate } & \multicolumn{3}{|c|}{ SD } & \multicolumn{3}{|c|}{ SE } \\
\hline & lixture & GTobit & Tobit & Mixture & GTobit & Tobit & Mixture & GTobit & Tobit \\
\hline & \multicolumn{9}{|c|}{$\eta=0.1$} \\
\hline$\eta$ & 100 & - & - & 0.007 & - & - & 0.008 & - & - \\
\hline$\alpha$ & - & 3.432 & 4.282 & - & 0.077 & 0.159 & - & 0.160 & 0.097 \\
\hline$\beta_{0}=1$ & 1.039 & 1.980 & 3.055 & 0.212 & 0.073 & 0.179 & 0.190 & 0.154 & 0.076 \\
\hline$\beta_{1}=-1$ & -1.060 & -0.205 & -0.811 & 0.210 & 0.036 & 0.038 & 0.190 & 0.040 & 0.035 \\
\hline$\gamma_{0}=1$ & 0.997 & 0.700 & - & 0.096 & 0.076 & - & 0.096 & 0.055 & - \\
\hline$\gamma_{1}=-1$ & -0.973 & -0.730 & - & 0.029 & 0.037 & - & 0.029 & 0.041 & - \\
\hline$\phi=4$ & 4.173 & 2.198 & - & 0.284 & 0.139 & - & 0.291 & 0.133 & - \\
\hline$\sigma^{2}=0.8$ & 0.826 & 0.586 & 0.549 & 0.094 & 0.082 & 0.087 & 0.094 & 0.120 & 0.081 \\
\hline \multirow[t]{2}{*}{$\rho=0.6$} & 0.573 & 0.566 & 0.561 & 0.063 & 0.066 & 0.070 & 0.066 & 0.115 & 0.071 \\
\hline & \multicolumn{9}{|c|}{$\eta=0.5$} \\
\hline$\eta$ & 0.502 & - & - & 0.012 & - & - & 0.013 & - & - \\
\hline$\alpha$ & - & 1.595 & 1.978 & - & 0.056 & 0.100 & - & 0.080 & 0.047 \\
\hline$\beta_{0}=1$ & 1.024 & 1.263 & 1.687 & 0.133 & 0.077 & 0.128 & 0.122 & 0.125 & 0.076 \\
\hline$\beta_{1}=-1$ & -1.012 & -0.389 & -0.607 & 0.081 & 0.033 & 0.034 & 0.084 & 0.033 & 0.035 \\
\hline$\gamma_{0}=1$ & 1.014 & 0.800 & - & 0.097 & 0.077 & - & 0.102 & 0.042 & - \\
\hline$\gamma_{1}=-1$ & -1.002 & -0.823 & - & 0.039 & 0.040 & - & 0.038 & 0.037 & - \\
\hline$\phi=4$ & 4.130 & 3.426 & - & 0.310 & 0.248 & - & 0.320 & 0.194 & - \\
\hline$\sigma^{2}=0.8$ & 0.826 & 0.326 & 0.329 & 0.111 & 0.080 & 0.082 & 0.107 & 0.142 & 0.079 \\
\hline \multirow[t]{2}{*}{$\rho=0.6$} & 0.573 & 0.558 & 0.555 & 0.069 & 0.087 & 0.089 & 0.070 & 0.229 & 0.093 \\
\hline & \multicolumn{9}{|c|}{$\eta=0.9$} \\
\hline$\eta$ & 0.901 & - & - & 0.008 & - & - & 0.008 & - & - \\
\hline$\alpha$ & - & 0.385 & 0.424 & - & 0.034 & 0.041 & - & 0.034 & 0.037 \\
\hline$\beta_{0}=1$ & 1.026 & 0.964 & 1.009 & 0.114 & 0.084 & 0.092 & 0.115 & 0.123 & 0.095 \\
\hline$\beta_{1}=-1$ & -1.008 & -0.702 & -0.729 & 0.062 & 0.046 & 0.045 & 0.066 & 0.047 & 0.042 \\
\hline$\gamma_{0}=1$ & 1.007 & 0.925 & - & 0.112 & 0.105 & - & 0.124 & 0.083 & - \\
\hline$\gamma_{1}=-1$ & -1.006 & -0.931 & - & 0.091 & 0.093 & - & 0.084 & 0.079 & - \\
\hline$\phi=4$ & 4.234 & 4.100 & - & 0.608 & 0.591 & - & 0.530 & 0.507 & - \\
\hline$\sigma^{2}=0.8$ & 0.817 & 0.459 & 0.470 & 0.133 & 0.141 & 0.141 & 0.137 & 0.187 & 0.124 \\
\hline$\rho=0.6$ & 0.573 & 0.566 & 0.565 & 0.078 & 0.102 & 0.101 & 0.080 & 0.202 & 0.100 \\
\hline
\end{tabular}


Table 4: Simulation study: composite likelihood estimates under the misspecified spatial beta-Bernoulli mixture model (Mixture) and spatial Tobit model (Tobit). The true model is the generalized spatial Tobit model (GTobit) with sample size $N=1600$ based on 100 simulations.

\begin{tabular}{r|rrrrrrrrr}
\hline \multirow{2}{*}{ Parameter } & \multicolumn{3}{|c}{ Estimate } & \multicolumn{3}{c}{ SD } & \multicolumn{3}{c}{ SE } \\
& Mixture & GTobit & Tobit & Mixture & GTobit & Tobit & Mixture & GTobit & Tobit \\
\hline$\alpha=3$ & - & 3.044 & 3.237 & - & 0.118 & 0.170 & - & 0.142 & 0.163 \\
$\eta$ & 0.316 & - & - & 0.017 & - & - & 0.012 & - & - \\
$\beta_{0}=1$ & -1.096 & 1.029 & 1.700 & 0.256 & 0.111 & 0.176 & 0.232 & 0.123 & 0.062 \\
$\beta_{1}=-1$ & -2.195 & -1.019 & -1.354 & 0.289 & 0.048 & 0.060 & 0.273 & 0.056 & 0.056 \\
$\gamma_{0}=1$ & 0.821 & 0.994 & - & 0.056 & 0.030 & - & 0.067 & 0.029 & - \\
$\gamma_{1}=-1$ & -1.622 & -0.995 & - & 0.044 & 0.043 & - & 0.045 & 0.044 & - \\
$\phi=4$ & 6.627 & 4.018 & - & 0.408 & 0.210 & - & 0.371 & 0.223 & - \\
$\sigma^{2}=0.8$ & 0.517 & 0.828 & 0.700 & 0.081 & 0.093 & 0.102 & 0.105 & 0.110 & 0.093 \\
$\rho=0.6$ & 0.559 & 0.573 & 0.565 & 0.078 & 0.063 & 0.068 & 0.092 & 0.080 & 0.068 \\
\hline
\end{tabular}


Table 5: Data example: maximum composite likelihood estimates (Est) and standard errors (SE) of the regression coefficients for intercept $\left(\beta_{0}, \gamma_{0}\right)$, reservation $\left(\beta_{1}, \gamma_{1}\right)$, number of parcels $\left(\beta_{2}, \gamma_{2}\right)$, proportion of the average parcel size $\left(\beta_{3}, \gamma_{3}\right)$, quarter section proportion of the largest parcel $\left(\beta_{4}, \gamma_{4}\right)$, average size of all parcels $\left(\beta_{5}, \gamma_{5}\right)$, log transformed total ownership area $\left(\beta_{6}, \gamma_{6}\right)$, and other parameters under the spatial beta-Bernoulli mixture model (Mixture), spatial generalized Tobit model (GTobit), spatial Tobit model (Tobit), and final models by backward elimination. Computing time is recorded in minutes.

\begin{tabular}{r|rr|rr|rr|rr|rr}
\hline \multirow{2}{*}{ Param } & \multicolumn{2}{|c|}{ Mixture } & \multicolumn{2}{|c|}{ Mixture Final } & \multicolumn{2}{|c|}{ GTobit } & \multicolumn{2}{|c}{ GTobit Final } & \multicolumn{2}{|c}{ Tobit } \\
& Est & SE & Est & SE & Est & SE & Est & SE & Est & SE \\
\hline$\alpha$ & - & - & - & - & 3.537 & 0.205 & 3.532 & 0.200 & 2.889 & 0.203 \\
$\eta$ & 0.121 & 0.009 & 0.121 & 0.009 & - & - & - & - & - & - \\
\hline$\beta_{0}$ & -1.143 & 0.273 & -1.113 & 0.238 & 1.245 & 0.152 & 1.242 & 0.152 & 1.169 & 0.119 \\
$\beta_{1}$ & 2.976 & 0.398 & 2.977 & 0.380 & 1.202 & 0.281 & 1.201 & 0.275 & 1.314 & 0.202 \\
$\beta_{2}$ & 0.024 & 0.265 & - & - & -0.024 & 0.112 & -0.012 & 0.065 & -0.020 & 0.074 \\
$\beta_{3}$ & -0.100 & 0.604 & - & - & 0.028 & 0.173 & - & - & -0.085 & 0.088 \\
$\beta_{4}$ & 0.183 & 0.479 & - & - & -0.050 & 0.138 & - & - & 0.121 & 0.088 \\
$\beta_{5}$ & 0.842 & 0.482 & 0.673 & 0.373 & 0.265 & 0.116 & 0.271 & 0.093 & 0.181 & 0.072 \\
$\beta_{6}$ & -0.286 & 0.452 & - & - & 0.023 & 0.086 & - & - & 0.154 & 0.077 \\
\hline$\gamma_{0}$ & -0.083 & 0.115 & -0.061 & 0.114 & 0.322 & 0.089 & 0.345 & 0.087 & - & - \\
$\gamma_{1}$ & 1.403 & 0.185 & 1.336 & 0.183 & 0.122 & 0.188 & 0.065 & 0.182 & - & - \\
$\gamma_{2}$ & -0.061 & 0.113 & - & - & -0.012 & 0.122 & -0.063 & 0.066 & - & - \\
$\gamma_{3}$ & -0.171 & 0.145 & - & - & -0.123 & 0.182 & - & - & - & - \\
$\gamma_{4}$ & 0.212 & 0.114 & - & - & 0.200 & 0.156 & - & - & - & - \\
$\gamma_{5}$ & 0.204 & 0.078 & 0.285 & 0.061 & -0.104 & 0.110 & -0.020 & 0.069 & - & - \\
$\gamma_{6}$ & 0.114 & 0.081 & - & - & 0.094 & 0.074 & - & - & - & - \\
\hline$\phi$ & 1.113 & 0.057 & 1.103 & 0.057 & 0.663 & 0.033 & 0.658 & 0.034 & - & - \\
$\sigma^{2}$ & 0.904 & 0.160 & 0.909 & 0.157 & 0.855 & 0.099 & 0.865 & 0.100 & 0.824 & 0.072 \\
$\rho$ & 0.502 & 0.081 & 0.503 & 0.080 & 0.641 & 0.078 & 0.637 & 0.078 & 0.647 & 0.060 \\
\hline CLIC & -0.662 & -0.676 & -0.641 & -0.688 & 1.827 & \\
Time & 27 & & 15 & & 28 & 20 & & 1 & \\
\hline
\end{tabular}

Table 6: Data example: mean absolute errors (MAE) and root mean squared errors (RMSE) computed for evaluating the prediction accuracy in training and test sets under the spatial beta-Bernoulli mixture model (Mixture), spatial generalized Tobit model (GTobit), and spatial Tobit model (Tobit).

\begin{tabular}{r|rr|rr}
\hline \multirow{2}{*}{ Model } & \multicolumn{2}{|c|}{ MAE } & \multicolumn{2}{c}{ RMSE } \\
& Training & Test & Training & Test \\
\hline Mixture & 0.096 & 0.188 & 0.137 & 0.249 \\
GTobit & 0.071 & 0.184 & 0.107 & 0.250 \\
Tobit & 0.096 & 0.198 & 0.122 & 0.240 \\
\hline
\end{tabular}

